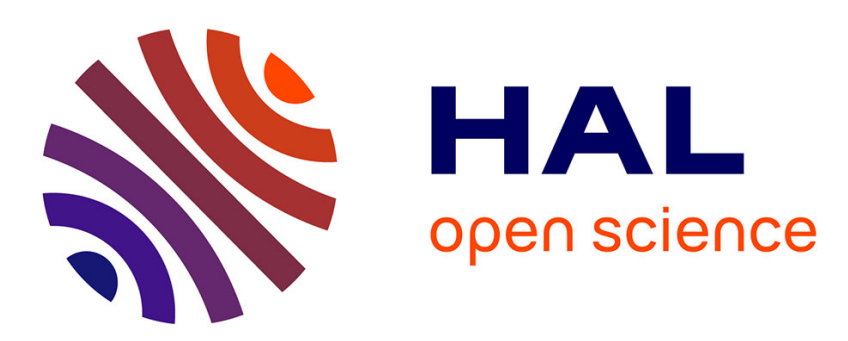

\title{
Multiscale modeling of skeletal muscle tissues based on analytical and numerical homogenization
}

\author{
L.A. A Spyrou, K. Danas, Sébastien Brisard
}

\section{To cite this version:}

L.A. A Spyrou, K. Danas, Sébastien Brisard. Multiscale modeling of skeletal muscle tissues based on analytical and numerical homogenization. Journal of the mechanical behavior of biomedical materials, 2019, 10.1016/j.jmbbm.2018.12.030 . hal-01965947

\section{HAL Id: hal-01965947 \\ https://hal-polytechnique.archives-ouvertes.fr/hal-01965947}

Submitted on 27 Dec 2018

HAL is a multi-disciplinary open access archive for the deposit and dissemination of scientific research documents, whether they are published or not. The documents may come from teaching and research institutions in France or abroad, or from public or private research centers.
L'archive ouverte pluridisciplinaire HAL, est destinée au dépôt et à la diffusion de documents scientifiques de niveau recherche, publiés ou non, émanant des établissements d'enseignement et de recherche français ou étrangers, des laboratoires publics ou privés. 


\title{
Multiscale modeling of skeletal muscle tissues based on analytical and numerical homogenization
}

\author{
L.A. Spyrou ${ }^{\mathrm{a}, *}$, S. Brisard ${ }^{\mathrm{b}}$, K. Danas ${ }^{\mathrm{c}}$ \\ ${ }^{a}$ Biomechanics Group, Institute for Bio-Economy $\&$ Agri-Technology, Centre for Research \& Technology Hellas (CERTH), \\ 38333 Volos, Greece \\ ${ }^{b}$ Université Paris-Est, Laboratoire Navier, UMR 8205, CNRS, ENPC, IFSTTAR, F-77455 Marne-la-Vallée, France \\ ${ }^{c} L M S, C N R S$, École Polytechnique, 91128 Palaiseau, France
}

\begin{abstract}
A novel multiscale modeling framework for skeletal muscles based on analytical and numerical homogenization methods is presented to study the mechanical muscle response at finite strains under three-dimensional loading conditions. First an analytical microstructure-based constitutive model is developed and numerically implemented in a general purpose finite element program. The analytical model takes into account explicitly the volume fractions, the material properties, and the spatial distribution of muscle's constituents by using homogenization techniques to bridge the different length scales of the muscle structure. Next, a numerical homogenization model is developed using periodic eroded Voronoi tessellation to virtually represent skeletal muscle microstructures. The eroded Voronoi unit cells are then resolved by finite element simulations and are used to assess the analytical homogenization model. The material parameters of the analytical model are identified successfully by use of available experimental data. The analytical model is found to be in very good agreement with the numerical model for the full range of loadings, and a wide range of different volume fractions and heterogeneity contrasts between muscle's constituents. A qualitative application of the model on fusiform and pennate muscle structures shows its efficiency to examine the effect of muscle fiber concentration variations in an organ-scale model simulation.
\end{abstract}

Key words: skeletal muscle, multiscale modeling, muscle mechanics, constitutive modeling, homogenization, finite element analysis

\section{Introduction}

Skeletal muscle has a complex hierarchical structure consisting of about 90-99\% force-producing fibers embedded in a connective tissue matrix. Skeletal muscles are distinct from other soft biological tissues due to their fibers' ability to produce force by active contraction. Each muscle fiber is surrounded by endomysium while a group of muscle fibers is surrounded by perimysium to form a muscle fascicle. The entire muscle volume is wrapped in an epimysial connective tissue covering. The intramuscular connective tissue (endomysium, perimysium, epimysium) accounts for about 1-10\% of a healthy skeletal muscle volume [24] and plays an important role in the muscle's functional characteristics, e.g. a) it adds to the passive elastic response of the muscle [58], and b) it has a major contribution to myo-fascial force transmission between neighboring fibers and fascicles [20].

On the other hand, muscle diseases (e.g. muscular dystrophy [56], spasticity [50]), large-strain muscle injuries [46], and immobilization [22] cause significant changes in the microstructural characteristics of muscle tissue (Fig. 1). Such microstructural changes may affect the macroscopic material behavior of the tissue. In

\footnotetext{
* Corresponding author.

Email addresses: 1.spyrou@certh.gr (L.A. Spyrou), sebastien.brisard@ifsttar.fr (S. Brisard), konstantinos.danas@polytechnique.edu (K. Danas)

Preprint accepted in Journal of Mechanical Behavior of Biomedical Materials
}

December 27, 2018 


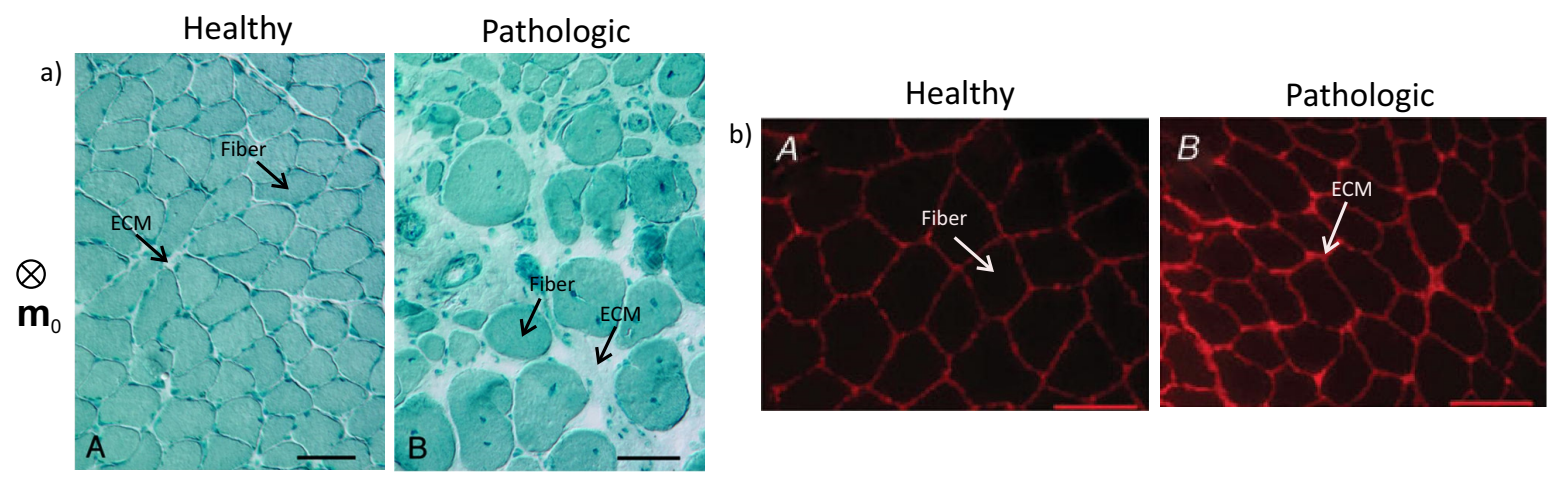

Figure 1: Comparison of histological cross-sections between healthy and pathologic muscle tissues affected by (a) Duchenne muscular dystrophy (scale bar represent $60 \mu \mathrm{m}$ ), and (b) cerebral palsy (scale bar represent $100 \mu \mathrm{m}$ ). In both cases the amount of ECM within diseased muscle tissue is increased. The unit vector along the muscle fiber direction in the undeformed configuration $\mathbf{m}_{0}$ is normal to the plane of the histological cross sections. (Adapted from Tidball and Wehling-Henricks [56] and Smith et al. [50] with permission.)

addition, regardless of their microstructural organization, both muscle fibers and connective tissue determine the overall properties of the whole muscle through their functional cooperation.

The physiological functioning of deformable soft tissues, such as skeletal muscles, crucially depends on their mechanical properties. In addition, an understanding of muscle properties can improve our ability to restore function when muscle force or movement is lost due to disease, trauma, or disuse [27]. Accordingly, many methods of clinical treatment and surgical interventions largely rely on muscle's mechanical effects [29]. Thus, state-of-the-art research and treatment of muscle tissues require highly accurate and efficient methods for describing their mechanical behavior and characterizing their mechanical response. Towards this direction, three-dimensional muscle tissue-level constitutive models have been developed based on the principles of continuum mechanics where muscle tissue is represented as a fiber-reinforced composite material [e.g. 3, 10, 52, 14]. Such models have been extensively used to investigate complex structural and functional aspects of skeletal muscle, such as stress-strain response, stress and strain distributions, and shape deformations of anatomical muscle structures in the context of finite element analysis [e.g. 26, 47, 4, 53].

However, current state-of-the-art continuum constitutive models for muscles generally are phenomenological in nature and they do not incorporate any microstructural information, i.e. the distinct contributions of the muscle constituents, such as their material properties, their geometrical characteristics, and their volume fractions in the tissue. Therefore, such models are unable to provide insight into skeletal muscle's macroscopic behavior upon its microstructural variations. To our knowledge, there is only the very recent work of Spyrou et al. [54] which proposed a constitutive model for skeletal muscles based on homogenization of the Voigt type in order to bridge the different length scales of the muscle structure. The Voigt estimate has the advantage of being especially simple, easy to compute, and for the special class of incompressible, transversely isotropic materials of being exact for axisymmetric loading conditions aligned with the symmetry axis of transverse isotropy (see, e.g., Agoras et al. [2]), which in the case of the skeletal muscle coincides with the direction of the fibers. However, for shear and in-plane normal loading conditions, the Voigt estimate is expected to be inaccurate, especially with increasing heterogeneity contrast between the properties of the constituents, due to its lack of adequate description of the underlying microstructure other than the volume fractions of the constituents. In order to overcome this shortcoming, in the present paper we make use of more sophisticated homogenization techniques which, in principle, deliver better estimates for the entire muscle's response under general loading conditions and a wide range of muscle fiber-ECM contrasts.

In addition to constitutive modeling, there are also very few studies presenting finite element (FE) modeling approaches considering skeletal muscle's microstructure. In particular, Ceelen et al. [5] developed a FE model of a transverse cross-section of skeletal muscle microstructure in order to study the deformationinduced hypoxic damage of muscle cells. Also, Sharafi and Blemker [48] created FE unit cells from histological cross-sections of rabbit muscles in order to derive the macroscopic along-fiber shear modulus of the skeletal 
muscle tissue by following a computational micromechanics approach. Further, Virgilio et al. [62] developed FE models of random skeletal muscle fascicle cross-sections based on an agent-based modeling framework in order to derive tissue-level mechanical properties upon disease-related microstructural changes. More recently, Marcucci et al. [35] used a histological-based FE model of a skeletal muscle fiber bundle composed of slow-and fast-twitch fibers to predict the bundle's effective force-velocity behavior.

The above discussion indicates that microstructure-based analytical or numerical modeling approaches for skeletal muscles are very limited in the literature. Further development of more sophisticated microstructurebased constitutive models that describe accurately the mechanical behavior of skeletal muscles is needed in order to be able to analyze 3D anatomical muscle structures while shedding light on how the macroscopic response of the skeletal muscle is affected by its micromechanical and microstructural properties. Also, more efficient methodologies in the context of computational micromechanics are required to be developed, in which the effective behavior of a muscle tissue could be obtained by FE analysis of a representative volume element (RVE) of its microstructure. Such developments become increasingly important for the better understanding of both healthy and diseased muscles' functional characteristics considering their significant structural variations among different subjects.

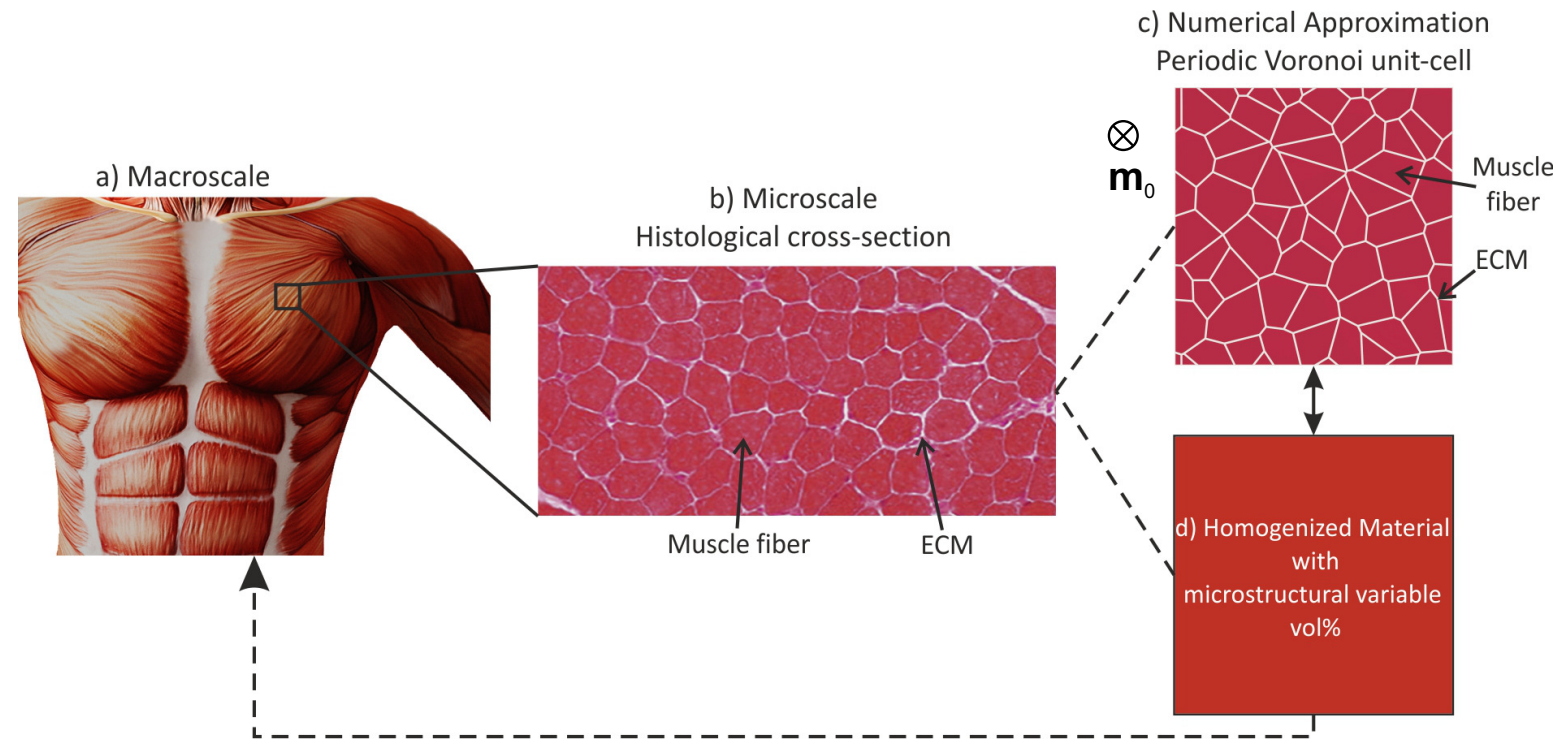

Figure 2: Schematic representation of the proposed multiscale modeling framework for skeletal muscles based on analytical and numerical homogenization. The unit vector along the muscle fiber direction in the undeformed configuration $\mathbf{m}_{0}$ is normal to the plane of the microstructural cross sections.

Towards this direction and by extending the previous work of Spyrou et al. [54], we propose, in this work, a novel multiscale modeling strategy for skeletal muscle structures based on analytical and numerical homogenization methods (Fig. 2). This strategy consists of two main tasks. The first task involves the development of an analytical homogenization constitutive model for the macroscopic response of skeletal muscle along with an algorithm for its numerical implementation in a general purpose finite element program. The second task considers a computational micromechanics methodology, which is used on one hand to obtain the effective mechanical behavior of various muscles numerically via FE simulations, and on the other hand to assess the accuracy of the analytical model. Inspired by histological cross-sections of skeletal muscle (see for instance Fig. 2b), the muscle structure can be numerically approximated by eroded Voronoi unit cells (see for instance Fig. 2c), where the Voronoi cells (i.e. polygons) represent the muscle fibers and the space between the cells represents the connective tissue surrounding the fibers.

More specifically, in Section 2 an analytical homogenization model for skeletal muscles that takes into account their microstructural characteristics (i.e., volume fractions, material properties, and spatial distribution of constituents) is introduced. Subsequently, Section 3 presents in detail a numerical homogenization 
model based on eroded Voronoi tessellations. The results of the study and the main features of the two models are presented and discussed in Section 4. In particular, the analytical model parameters are identified using experimental data from the literature and then comparisons are carried out among the analytical model predictions and the numerical model estimates under the full range of 3D loading conditions, a wide range of muscle fiber volume fractions (FVF from $60 \%$ to $90 \%$ ), and a wide range of heterogeneity contrast between muscle fibers and intramuscular connective tissue (shear modulus contrast from 5 to 50). Finally, to show the usefulness of such models in the context of macroscale muscle geometries, the analytical model is applied on idealized fusiform and pennate muscle structures in order to examine qualitatively their mechanical response upon microstructural changes.

Standard notation is used throughout. Boldface symbols denote tensors the orders of which are indicated by the context. The usual summation convention is used for repeated Latin indices of tensor components with respect to a fixed Cartesian coordinate system with base vectors e $(i=1,2,3)$. The prefaces "det" and "tr" indicate the determinant and trace respectively, a superscript $T$ the transpose, and a superposed dot the material time derivative. Let $\mathbf{a}, \mathbf{b}, \mathbf{c}, \mathbf{d}$ be vectors, $\boldsymbol{A}, \boldsymbol{B}$ second-order tensors, and $\mathcal{C}, \mathcal{D}$ fourth-order tensors; the following products are used in the text: $(\mathbf{a b})_{i j}=a_{i} b_{j},(\mathbf{a b c d})_{i j k l}=a_{i} b_{j} c_{k} d_{l},(\mathbf{a} \cdot \boldsymbol{A})_{i}=a_{j} A_{j i}$, $(\boldsymbol{A} \cdot \mathbf{a})_{i}=A_{i j} a_{j},(\mathbf{a} \cdot \boldsymbol{A} \cdot \mathbf{a})_{i j}=a_{i} A_{i j} a_{j},(\mathbf{a} \boldsymbol{A} \cdot \mathbf{a})_{i j}=a_{i} A_{j k} a_{k},(\mathbf{a} \cdot \boldsymbol{A} \mathbf{a})_{i j}=a_{k} A_{k i} a_{j}, \boldsymbol{A} \cdot \boldsymbol{B}=A_{i k} B_{k j}$, $\boldsymbol{A}: \boldsymbol{B}=A_{i j} B_{i j},(\boldsymbol{A}: \mathcal{C})_{i j}=A_{k l} C_{k l i j},(\mathcal{C}: \boldsymbol{A})_{i j}=C_{i j k l} A_{k l}$, and $(\mathcal{C}: \mathcal{D})_{i j k l}=C_{i j p q} D_{p q k l}$

\section{Analytical homogenization model}

Skeletal muscles may be viewed as composite materials consisting of muscle fibers embedded in a matrix of intramuscular connective tissue (endomysium, perimysium, and epimysium). The hierarchical nature of a skeletal muscle's structure makes it possible to employ a three-scale homogenization scheme for the development of a microstructure-based tissue-level constitutive model. The first scale includes the homogenization of a muscle fascicle's behavior considering the individual properties of muscle fibers and endomysium. The second scale includes the homogenization of the whole muscle's behavior (epimysium not included) taking into account the individual properties of perimysium and muscle fascicles as resulted from the first scale. Finally, the contribution of the epimysial connective tissue on the whole muscle macroscopic behavior can be taken into account in a third scale homogenization model. However, in this work, due to non-availability of enough distinct microstructural data (i.e. mechanical properties, morphological characteristics, volume fractions) separately associated with endomysium, perimysium, and epimysium, we choose to lump the material properties of these three types of connective tissue, collectively consider them as one single structure named extracellular matrix (ECM), and make use of a single-scale homogenization framework as depicted in Fig. 2 .

Thus, we model skeletal muscle as a two-phase composite material made out of a large number of homogeneous muscle fibers (phase 1), surrounded by and perfectly bonded to a homogeneous ECM (phase 2 ). The fibers are further assumed to be aligned along a given direction $\mathbf{m}_{0}$ in the undeformed configuration and distributed in the transverse plane. The details concerning the constitutive relations characterizing the material behavior of the constituent phases are given in subsection 2.1, while the homogenization procedure used to obtain the macroscopic or homogenized behavior of the composite skeletal muscle is discussed in subsection 2.2 .

\subsection{Constitutive relations for the phases}

A muscle fiber is composed of a bundle of many myofibrils arranged in parallel and embedded in a matrix of sarcoplasm. In the same sense, ECM is composed of networks of collagen fibers embedded in a matrix of proteoglycans; these collagen networks show generally preferred orientations (with respect to the long axis of the muscle fibers) which change with changing muscle length [42, 43]. Therefore, and following earlier work [e.g. 48, 49, 62, 54], we model the ECM and the muscle fibers as two different, nearly incompressible, transversely isotropic solids, characterized by the same symmetry axis $\mathbf{m}_{0}$ in the undeformed configuration. In particular, we assume that the total true stress tensor $\boldsymbol{\sigma}^{(r)}$ at any given material point $\mathbf{X}$ in the continuum 
may be written as the sum of an isotropic part $\boldsymbol{\sigma}_{i}^{(r)}$ and an anisotropic part $\boldsymbol{\sigma}_{a}^{(r)}$ :

$$
\boldsymbol{\sigma}^{(r)}=\boldsymbol{\sigma}_{i}^{(r)}+\boldsymbol{\sigma}_{a}^{(r)}
$$

where the superscript $r=1$ when $\mathbf{X}$ is in the fiber phase and $r=2$ when $\mathbf{X}$ is in the ECM phase. This decomposition is a constitutive assumption [17] that is able to deliver simple but also very accurate results by comparison with available experiments (as discussed later in Fig.7). The anisotropic part $\boldsymbol{\sigma}_{a}^{(r)}$ of the stress tensor in (1) is associated with the fibrous part of the constituents and represents the stress response in the muscle fiber direction. In turn, $\boldsymbol{\sigma}_{i}^{(r)}$ is associated with the non-fibrous part of the constituents and mainly represents the response under shear loading and transverse normal straining with respect to the material's preferred direction. In particular, as regards to the ECM, the isotropic part is associated with the behavior of the matrix of proteoglycans whereas the anisotropic part considers the mechanical contribution of the collagen fibers. As regards to the muscle fiber, the anisotropic part describes the behavior of the fiber's contractile structure whereas the isotropic part is associated with the contribution of all the rest membrane structures and biofluids contained in a muscle fiber.

We assume in the present study that the isotropic part can be fairly well described by a simple nonlinear energy density. Subsequently, we provide specific constitutive equations for $\boldsymbol{\sigma}_{i}^{(r)}$ and $\boldsymbol{\sigma}_{a}^{(r)}$.

The isotropic part of the stress $\boldsymbol{\sigma}_{i}^{(r)}$ in (1) is assumed to be of the hyperelastic form

$$
\boldsymbol{\sigma}_{i}^{(r)}=\frac{2}{J} \boldsymbol{F} \frac{\partial W^{(r)}}{\partial \boldsymbol{C}} \boldsymbol{F}^{T}
$$

where $\boldsymbol{F}$ stands for the deformation gradient at point $\mathbf{X}, J=\operatorname{det} \boldsymbol{F}, \boldsymbol{C}=\boldsymbol{F}^{T} \boldsymbol{F}$ is the right Cauchy-Green deformation tensor and $W^{(r)}$ is the associated stored energy function of phase $r$. It is common practice to describe the isotropic behavior of soft biological tissues [e.g. 17] by use of Neo-Hookean models, such as

$$
W^{(r)}(\boldsymbol{F})=\frac{G^{(r)}}{2}\left(\bar{I}_{1}-3\right)+\frac{K^{(r)}}{2}(J-1)^{2} .
$$

Here, $\bar{I}_{1}=J^{-2 / 3} I_{1}$, with $I_{1}=\operatorname{tr} \boldsymbol{C}$, while the constants $G^{(r)}$ and $K^{(r)}$ are respectively the shear and bulk moduli of phase $r$. Making use of expression (3) in (2), it can be easily shown that

$$
\boldsymbol{\sigma}_{i}^{(r)}=\frac{G^{(r)}}{J}\left(\bar{B}-\frac{1}{3} \operatorname{tr}(\overline{\boldsymbol{B}}) \boldsymbol{\delta}\right)+K^{(r)}(J-1) \boldsymbol{\delta},
$$

where $\overline{\boldsymbol{B}}=J^{-2 / 3} \boldsymbol{B}$, with $\boldsymbol{B}=\boldsymbol{F} \boldsymbol{F}^{T}$ denoting the left Cauchy-Green deformation tensor, and $\boldsymbol{\delta}$ is the second-order identity tensor. Note that, incompressible Neo-Hookean behavior corresponds to the limiting case $K^{(r)} \longrightarrow \infty$, along with the constraint $J=1$, in (3) and (4). In the present study, we retain a very small compressible part but consider that $K^{(r)} \gg G^{(r)}(r=1,2)$. This approximation does not alter the final results and is convenient for the numerical implementation of the presented models.

The anisotropic part of the stress $\boldsymbol{\sigma}_{a}^{(r)}$ in (1) is assumed to be of the form [52]

$$
\boldsymbol{\sigma}_{a}^{(r)}=\sigma^{(r)} \mathbf{m} \mathbf{m}, \quad \mathbf{m}=\frac{1}{\left|\boldsymbol{F} \cdot \mathbf{m}_{0}\right|} \boldsymbol{F} \cdot \mathbf{m}_{0} .
$$

In this expression, $\mathbf{m}$ is the unit vector along the transversely isotropic symmetry axis in the deformed configuration, whereas $\sigma^{(r)}$ is the current axial stress, and is given in terms of the reference axial stress $\sigma_{0}^{(r)}$ by [54]

$$
\sigma^{(r)}=\sigma_{0}^{(r)} \lambda_{m},
$$

with $\lambda_{m}$ denoting the axial stretch ratio in the muscle fiber direction. We note that $\sigma_{0}^{(r)}$ is not a constant and is different for the muscle fibers $(r=1)$ and the ECM $(r=2)$ as detailed in the following subsections. 


\subsubsection{Anisotropic stress $\sigma_{0}^{(1)}$ for the muscle fibers}

Muscle fibers exhibit both "active" and "passive" mechanical characteristics. We consider that the nominal longitudinal stress in a muscle fiber can be written as the sum of an active and a passive part, such that

$$
\sigma_{0}^{(1)}=\sigma_{0}^{(1, a c t)}+\sigma_{0}^{(1, p a s)} .
$$

The active behavior is known to depend on the fiber length and velocity of contraction as well as on the activation level of the muscle, whereas the passive behavior depends mainly on the fiber length. From a "continuum" point of view, this means that the muscle fiber stress depends on the local stretch ratio, stretch-rate, and activation level. Following Van Leeuwen and Kier [59], we write

$$
\sigma_{0}^{(1, a c t)}=\sigma_{\max } f_{a} f_{e}\left(\lambda_{m}\right) f_{r}\left(\dot{\lambda}_{m}\right), \quad \sigma_{0}^{(1, p a s)}=f_{p}^{(1)}\left(\lambda_{m}\right),
$$

where $\sigma_{\max }$ is the maximum isometric stress that appears at optimal fiber length, $f_{a}$ is the activation function that controls the magnitude of $\sigma_{0}^{(1, a c t)}, f_{e}$ describes the dependence of the active stress on the axial stretch $\lambda_{m}, f_{r}$ is the function that relates the active muscle stress to the axial stretch rate $\dot{\lambda}_{m}$, and $f_{p}^{(1)}$ describes the dependence of the passive stress on the axial stretch $\lambda_{m}$. In the above equation functions $\left(f_{a}, f_{e}, f_{r}\right)$ are dimensionless and normalized so that

$$
0 \leq f_{a} \leq 1, \quad \max _{\lambda_{m}} f_{e}\left(\lambda_{m}\right)=1, \quad f_{r}(0)=1 .
$$

The active function $f_{e}$ is described by a normalized Weibull distribution with two parameters $\lambda_{m}^{\min }$ and $\lambda_{m}^{\mathrm{opt}}$ as proposed in Ehret et al. [10]

$$
f_{e}\left(\lambda_{m}\right)= \begin{cases}\frac{\lambda_{m}^{\mathrm{min}}-\lambda_{m}}{\lambda_{m}^{\mathrm{min}}-\lambda_{m}^{\mathrm{opt}}} \exp \left[\frac{\left(2 \lambda_{m}^{\mathrm{min}}-\lambda_{m}-\lambda_{m}^{\mathrm{opt}}\right)\left(\lambda_{m}-\lambda_{m}^{\mathrm{opt}}\right)}{2\left(\lambda_{m}^{\mathrm{min}}-\lambda_{m}^{\mathrm{opt}}\right)^{2}}\right] & \text { if } \lambda_{m}>\lambda_{m}^{\min }, \\ 0 & \text { otherwise }\end{cases}
$$

where $\lambda_{m}^{\min }$ denotes the minimum fiber stretch at which muscle fiber begins to generate force, and $\lambda_{m}^{\text {opt }}$ is the optimal fiber stretch, i.e. the fiber stretch at which muscle fiber reaches optimal length and thus the maximum force is generated $[3,65]$.

The well-known hyperbolic function $f_{r}$ is used to describe the stretch rate dependence on the muscle fiber stress $[4,59]$

$$
f_{r}\left(\dot{\lambda}_{m}\right)= \begin{cases}d-(d-1) \frac{1+t^{*}}{1-k_{c} k_{e} t^{*}} & \text { if } t^{*}<0, \\ \frac{1-t^{*}}{1+k_{c} t^{*}} & \text { if } 0 \leq t^{*} \leq 1 \\ 0 & \text { if } t^{*}>1\end{cases}
$$

where $t^{*}=\dot{\lambda}_{m} / \dot{\lambda}_{m}^{\text {min }}, \dot{\lambda}_{m}^{\text {min }}<0$ is the minimum stretch rate, and $k_{c}, k_{e}, d$ are dimensionless material constants with $k_{c}, k_{e}>0$ and $d>1$, which guarantee that $f_{r}$ is positive with a positive slope in the range of application, i.e. $t^{*} \leq 1$.

Recent experimental data have shown a linear stress-strain passive behavior of single muscle fibers [37, 50], thus the muscle fiber passive stress-strain relation $f_{p}^{(1)}$ can be described by a linear function of fiber stretch ratio as

$$
f_{p}^{(1)}\left(\lambda_{m}\right)= \begin{cases}E_{p}\left(\lambda_{m}-\lambda_{m}^{\mathrm{opt}}\right) & \text { if } \lambda_{m}>\lambda_{m}^{\mathrm{opt}} \\ 0 & \text { otherwise }\end{cases}
$$



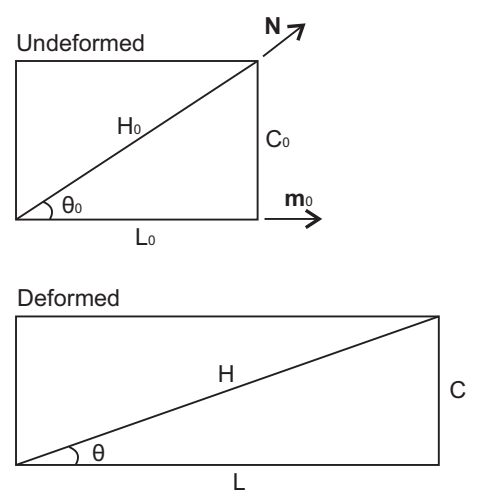

(a)

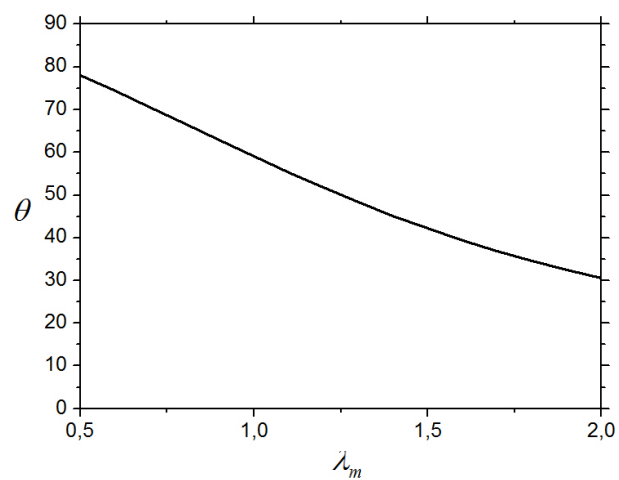

(b)

Figure 3: (a) A flattened schematic representation of a helical ECM fibril surrounding a cylindrical muscle fiber before and after imposing a stretch in the muscle fiber direction. (b) Relationship between the muscle fiber stretch and the angle $\theta$ between the collagen helix and the muscle fiber.

where $E_{p}$ denotes the muscle fiber's passive elastic modulus. It is noted that, in general, a linear behavior is experimentally observed for sarcomere lengths above $2.5 \mu \mathrm{m}$ [50]. At lower sarcomere lengths the behavior may present a short nonlinear "toe region" [45], however most fibers are generating tension beyond that region, thus a toe region has not been included in Eq. (12).

The various constants in the previous expressions are defined by use of experimental data in Section 4.1.

\subsubsection{Anisotropic stress $\sigma_{0}^{(2)}$ for the ECM}

The ECM's collagenous fiber network develops only "passive" stresses and, in accord with experimental studies, microscopically possesses a preferred orientation around $55-60$ degrees with respect to the muscle fiber direction [42, 43] at muscle's rest length. Following Purslow [42], we assume that the ECM's fiber network represents a helical fiber distribution with a preferred angle wrapped around incompressible cylindrical muscle fibers and that these collagen fibril helices are constrained to deform with the muscle fibers so that the stretch of a collagen helix $\lambda_{H}$ can be expressed in terms of the stretch of a muscle fiber $\lambda_{m}$ and the collagen fibril angle with respect to the muscle fiber at rest length $\theta_{0}$ as

$$
\lambda_{H}=\sqrt{\mathbf{N} \cdot \boldsymbol{C} \cdot \mathbf{N}}=\frac{H}{H_{0}}=\sqrt{\lambda_{m}{ }^{2} \cos ^{2} \theta_{0}+\frac{1}{\lambda_{m}} \sin ^{2} \theta_{0}}, \quad \lambda_{m}=\sqrt{\mathbf{m}_{0} \cdot \boldsymbol{C} \cdot \mathbf{m}_{0}} .
$$

Here, in correspondence with Fig. 3a, $\mathbf{N}$ denotes the unit vector in the direction of a collagen fibril in the reference configuration, and $H_{0}=\sqrt{{L_{0}}^{2}+C_{0}{ }^{2}}, H=\sqrt{L^{2}+C^{2}}$ denote the length of the collagen helix in the undeformed and deformed configuration respectively. In the last expressions, $C_{0}=2 \pi R_{0}$ and $C=2 \pi R$ denote the circumference of a muscle fiber of radius $\left(R_{0}, R\right)$ and length $\left(L_{0}, L\right)$ before and after imposing a stretch in the muscle fiber direction respectively. One can also derive a relation connecting the applied stretch $\lambda_{m}$ and the angle $\theta$ between the collagen helix and the muscle fiber in the deformed configuration, such that

$$
\tan \theta=\frac{C}{L}=\frac{\tan \theta_{0}}{\lambda_{m} \sqrt{\lambda_{m}}}
$$

Note that, in (14) use has been made of the incompressibility constraint $\pi R_{0}{ }^{2} L_{0}=\pi R^{2} L$. An illustration of the aforementioned relationship is depicted in Fig. $3 \mathrm{~b}$ for an initial angle $\theta_{0}=59^{\circ}$; the resulting straininduced reorientation behavior of an ECM collagen fiber is consistent with the experimental measurements of Purslow [42] and Purslow and Trotter [43].

In turn, the total nominal fiber stress of ECM can be written as a function of the stretch in the muscle fiber direction and the declination angle between the collagen fibrils and the muscle fiber

$$
\sigma_{0}^{(2)}=f_{p}^{(2)}\left(\lambda_{H}\right)=f_{p}^{(2)}\left(\lambda_{m}, \theta_{0}\right) .
$$


An exponential function $f_{p}^{(2)}$ is adopted here to describe the mechanical behavior of the collagenous connective tissue [17]

$$
f_{p}^{(2)}\left(\lambda_{H}\right)= \begin{cases}A 1\left(e^{A 2\left(\lambda_{H}-1\right)}-1\right) & \text { if } \lambda_{H}>1, \\ 0 & \text { otherwise. }\end{cases}
$$

Here, $A 1$ and $A 2$ are material coefficients. In deriving the last equation, we have taken into account that the fibrous parts of the muscle's constituents do not support passive compressive loads (see e.g. Holzapfel and Ogden [19])

\subsection{Analytical homogenization estimates for the macroscopic behavior}

In this section, we combine the response of the ECM and muscle fiber constituents in an effort to propose an analytical "approximate" mathematical model for the effective macroscopic response of the ensemble. The goal is to obtain a simple but accurate model that can be easily implemented in a user material routine for larger scale simulations of real muscle geometries (see diagram in Fig. 2).

Let us consider, then, a representative volume element (RVE) of a composite skeletal muscle material, as defined above, which in the undeformed configuration occupies a region $V_{0}$ with boundary $\partial V_{0}$, and let $V_{0}^{(1)}$ and $V_{0}^{(2)}$ be the complementary parts of $V_{0}$ occupied by muscle fibers (phase 1) and ECM (phase 2), respectively. Then, the volume fractions of the muscle fibers and the ECM in the RVE are

$$
\frac{\left|V_{0}^{(1)}\right|}{\left|V_{0}\right|} \equiv c \equiv \mathrm{FVF}, \quad \frac{\left|V_{0}{ }^{(2)}\right|}{\left|V_{0}\right|} \equiv 1-c,
$$

respectively. The particular shape of $\partial V_{0}$ is not important in the present context since we consider that the size of the RVE is much larger than the size of the heterogeneity, i.e., the diameter of a typical fiber.

The homogenization problem for the composite material of interest may be defined by means of affine boundary conditions $[15,41]$

$$
\mathbf{x}(t)=\widehat{\boldsymbol{F}}(t) \mathbf{X} \quad \forall \quad \mathbf{X} \quad \in \quad \partial V_{0},
$$

where $\mathbf{X}$ and $\mathbf{x}(t)$ denote the position vectors of any given material point on the boundary of the body in the undeformed and deformed (at time $t$ ) configuration, respectively, while $\widehat{\boldsymbol{F}}(t)$ is a deformation gradient tensor which may vary with time $t$, but is independent of $\mathbf{X}$, i.e., $\widehat{\boldsymbol{F}}(t)$ is constant for given $t$. Note that, due to the almost incompressible response of the material, the volume fractions of the phases (17) are independent of the deformation of the body. Letting $\boldsymbol{F}(\mathbf{X}, t)$ be the (unknown) deformation gradient field produced in the RVE under the boundary conditions (18), one can easily show that

$$
\widehat{\boldsymbol{F}}(t)=\frac{1}{\left|V_{0}\right|} \int_{V_{0}} \boldsymbol{F}(\mathbf{X}, t) \mathrm{d} \mathbf{X},
$$

i.e., the average of $\boldsymbol{F}(\mathbf{X}, t)$ over the RVE reference volume $V_{0}$ is equal to the applied deformation gradient $\widehat{\boldsymbol{F}}(t)$. For convenience and latter reference, we also introduce at this point the notation

$$
\widehat{\boldsymbol{\sigma}}(t)=\frac{1}{|V|} \int_{V} \boldsymbol{\sigma}(\mathbf{x}, t) \mathrm{d} \mathbf{x}
$$

for the average of the corresponding (unknown) true stress field $\boldsymbol{\sigma}(\mathbf{x}, t)$ over the region $V$ occupied by the body in the deformed (at time $t$ ) configuration.

The macroscopic or homogenized behavior of the skeletal muscle may then be defined by the relation between the average stress $\widehat{\boldsymbol{\sigma}}(t)$ and the history of the average deformation of the RVE, as prescribed by the deformation gradient $\widehat{\boldsymbol{F}}(t)$.

Guided by the decomposition (1) for $\boldsymbol{\sigma}^{(r)}$ and in order to derive a tractable analytical but also accurate model, we propose an approximate decoupled homogenization strategy. This consists in writing the macroscopic average Cauchy (Eulerian) stress $\widehat{\boldsymbol{\sigma}}(t)$ as a sum of a stress resulting from the homogenization of the 
constituents' isotropic behavior $\widehat{\boldsymbol{\sigma}}_{i}(t)$ and a stress resulting from the homogenization of the constituents' anisotropic behavior $\widehat{\boldsymbol{\sigma}}_{a}(t)$, i.e.,

$$
\widehat{\boldsymbol{\sigma}}(t)=\widehat{\boldsymbol{\sigma}}_{i}(t)+\widehat{\boldsymbol{\sigma}}_{a}(t) .
$$

In this regard and based on a previous work [54] where the Voigt approximation was used to estimate the homogenized behavior of the skeletal muscle and taking into account that the Voigt estimate is exact for axisymmetric loading conditions aligned with the direction of the muscle fibers, the anisotropic stress $\widehat{\boldsymbol{\sigma}}_{a}(t)$ in $(21)$ is given by

$$
\widehat{\boldsymbol{\sigma}}_{a}(t)=\widehat{\sigma}(t) \widehat{\mathbf{m}}(t) \widehat{\mathbf{m}}(t), \quad \widehat{\mathbf{m}}(t)=\frac{1}{\left|\widehat{\boldsymbol{F}}(t) \cdot \mathbf{m}_{0}\right|} \widehat{\boldsymbol{F}}(t) \cdot \mathbf{m}_{0}, \quad \widehat{\sigma}(t)=\widehat{\sigma}_{0}(t) \widehat{\lambda}_{m}(t),
$$

where we recall that $\mathbf{m}_{0}$ is the unit vector along the transversely isotropic symmetry axis in the undeformed configuration, $\widehat{\lambda}_{m}(t)$ denotes the stretch ratio induced by $\widehat{\boldsymbol{F}}(t)$ along the direction $\mathbf{m}_{0}$, and

$$
\widehat{\sigma}_{0}(t)=c \sigma_{0}^{(1)}\left(\widehat{\lambda}_{m}(t), \dot{\hat{\lambda}}_{m}(t)\right)+(1-c) \sigma_{0}^{(2)}\left(\widehat{\lambda}_{m}(t)\right)
$$

is the effective reference axial stress of the composite. Note that, in the above expression (23) the phase response functions $\sigma_{0}^{(r)}$, with $r=1,2$, defined in the previous subsection, are evaluated at $\widehat{\lambda}_{m}(t)$ and $\dot{\hat{\lambda}}_{m}(t)$. Again, the mapping of the reference vector $\mathbf{m}_{0}$ to $\widehat{\boldsymbol{F}}(t) \cdot \mathbf{m}_{0}$ in the deformed configuration, constitutes a rigorous result of homogenization [33] implying that long fibers behave as macroscopic material line elements. This result, in turn, supports the assumption adopted in phenomenological theories to treat fibers as material line elements that deform in an affine manner.

In turn, the isotropic part requires a more elaborate homogenization approach. Specifically, deBotton [7], deBotton et al. [8] and Lopez-Pamies and Idiart [32] have made use of solutions for isotropic coated (or high-rank) laminates made of hyperelastic constituents. Those estimates have been used recently by López Jímenez [30] in the context of iterated homogenization methods (see for instance Lopez-Pamies [31] for nonlinear hyperelastic composites) to produce estimates for two-phase hyperelastic composites comprising fibers of circular cross-section. Those estimates were assessed by numerical RVE calculations of random dispersion of fibers and were found to give a very good agreement up to volume fractions of $c=50 \%$. In the present work, the proposed microstructures exhibit two main differences; (i) the fiber volume fraction is extremely high ranging from $c=60 \%$ to at least $95 \%$ and (ii) the fiber cross-section is not circular but polygonal (see Fig. 2). Even so, the overall response is almost isotropic in the plane and as we will see the model of Lopez-Pamies (see López Jímenez [30]) in an otherwise different context delivers extremely accurate estimates. It is perhaps relevant to note here that in a large number of studies the response of the muscle is considered isotropic in the transverse plane of the fibers. As we will see later, this requires a sufficiently large volume to attain representativity and isotropy. On the other hand, in several numerical studies (see for instance Sharafi and Blemker [48]) the use of a direct experimentally-obtained image can lead to anisotropic in-plane response mainly due to the relatively small size of the volume considered and consequently its strong dependence upon the applied boundary conditions. These issues are considered and discussed in detail henceforth, especially in the numerical model of the following section.

Specifically, following closely López Jímenez [30], the effective stored-energy function depends on $\left(\widehat{\widehat{I}}_{1}, \widehat{I}_{4}, \widehat{I}_{5}, \widehat{J}\right)$ and reads as follows

$$
\widehat{W}_{I H}=\left[\frac{G_{I H}}{2}\left(\widehat{\widehat{I}}_{1}-3\right)+\frac{G_{n}-G_{I H}}{2}\left(\frac{2}{\sqrt{\widehat{I}_{4}}}-3\right)+\frac{G_{n}-G_{H S}}{2} \widehat{I}_{4}-\frac{G_{I H}-G_{H S}}{2} \frac{\widehat{I}_{5}}{\widehat{I}_{4}}\right]+\frac{\widehat{K}}{2}(\widehat{J}-1)^{2},
$$


where

$$
\begin{aligned}
& {\widehat{I_{1}}}_{1}=\widehat{J}^{-2 / 3} \widehat{I}_{1}=\widehat{J}^{-2 / 3} \operatorname{tr} \widehat{\boldsymbol{C}}=\widehat{J}^{-2 / 3} \operatorname{tr}\left(\widehat{\boldsymbol{F}}^{\mathrm{T}} \cdot \widehat{\boldsymbol{F}}\right), \\
& \widehat{I}_{4}=\mathbf{m}_{0} \cdot \widehat{\boldsymbol{C}} \cdot \mathbf{m}_{0}=\widehat{\lambda}_{m}^{2}, \\
& \widehat{I}_{5}=\mathbf{m}_{0} \cdot \widehat{\boldsymbol{C}}^{2} \cdot \mathbf{m}_{0}, \\
& G_{I H}=(1-c)^{2}\left(1+\frac{2(2-c) c G^{(1)}}{(1-c)^{2}} \frac{G^{(1)^{2}}}{G^{(2)}}\right) \frac{G^{(2)}}{G^{(2)^{2}}}- \\
& -(1-c)^{2} \frac{G^{(1)}-G^{(2)}}{2} \sqrt{\frac{2}{(1-c)^{2}} \frac{G^{(1)}}{G^{(2)}}+\left(1+\frac{2(2-c) c}{(1-c)^{2}} \frac{G^{(1)}}{G^{(2)}}+\frac{G^{(1)^{2}}}{G^{(2)^{2}}}\right)} \\
& G_{n}=c G^{(1)}+(1-c) G^{(2)}, \\
& G_{H S}=\frac{(1-c) G^{(2)}+(1+c) G^{(1)}}{(1+c) G^{(2)}+(1-c) G^{(1)}} G^{(2)} \\
& \widehat{K}=c K^{(1)}+(1-c) K^{(2)} .
\end{aligned}
$$

The proposed model in the present study differs from that of López Jímenez [30] only in terms of the augmented with $\widehat{J}$ invariant $\widehat{\widehat{I}}_{1}=\widehat{J}^{-2 / 3} \widehat{I}_{1}$ and the compressibility part involving the effective bulk modulus $\widehat{K}$. The latter is introduced by simple use of a Voigt homogenization and constitutes an approximation. The dependence upon $\widehat{J}$ can be readily dropped to deal with exact incompressibility in the limit of $K^{(1)}, K^{(2)} \longrightarrow$ $\infty$ or equivalently $\widehat{K} \longrightarrow \infty$, since the model in (24) has been originally defined in that limit. Nevertheless, for convenience in the numerical implementation in our present study, we use this term to avoid introducing the pressure as an additional Lagrange multiplier and use of non-standard finite element formulations. We make sure however that $K^{(r)} \gg G^{(r)}(r=1,2)$ for both constituents.

The stress $\widehat{\boldsymbol{\sigma}}_{i}(t)$ in relation $(21)$ is then given by

$$
\begin{aligned}
\widehat{\boldsymbol{\sigma}}_{i}(t) & =\frac{2}{\widehat{J}} \widehat{\boldsymbol{F}} \frac{\partial \widehat{W}_{I H}}{\partial \widehat{\boldsymbol{C}}} \widehat{\boldsymbol{F}}^{T}=\widehat{K}(\widehat{J}-1) \boldsymbol{\delta}+\frac{G_{I H}}{\widehat{J}}\left(\widehat{\widehat{\boldsymbol{B}}}-\frac{1}{3} \widehat{I}_{1} \boldsymbol{\delta}\right) \\
& +\frac{1}{\widehat{J}}\left(\frac{G_{I H}-G_{n}}{\sqrt{\widehat{I}_{4}}}+\frac{\left(G_{I H}-G_{H S}\right) \widehat{I}_{5}}{\widehat{I}_{4}}+\widehat{I}_{4}\left(G_{n}-G_{H S}\right)\right) \widehat{\mathbf{m}} \widehat{\mathbf{m}}-\frac{G_{I H}-G_{H S}}{\widehat{J}}(\widehat{\mathbf{m}} \widehat{\boldsymbol{B}} \cdot \widehat{\mathbf{m}}+\widehat{\mathbf{m}} \cdot \widehat{\boldsymbol{B}} \widehat{\mathbf{m}})
\end{aligned}
$$

where $\widehat{J}=\operatorname{det} \widehat{\boldsymbol{F}}(t), \widehat{\widehat{\boldsymbol{B}}}(t)=\widehat{J}^{-2 / 3} \widehat{\boldsymbol{B}}(t)$, with $\widehat{\boldsymbol{B}}(t)=\widehat{\boldsymbol{F}}(t) \widehat{\boldsymbol{F}}^{T}(t)$.

The constitutive model developed in this section has been implemented in the ABAQUS general-purpose finite element program [1]. ABAQUS provides a general interface so that a particular constitutive model can be introduced as a "user subroutine" (UMAT). A detailed description of the numerical implementation of the mathematical model is provided in the Appendix A.

\section{Numerical homogenization model}

In this section we present a numerical homogenization approach using a sufficiently complex three dimensional periodic unit cell. Inspired by skeletal muscle histological cross-sections (see for e.g. [49]) a muscle structure may be numerically approximated by eroded Voronoi unit-cells. The purpose of this numerical homogenization framework is twofold. First, the numerical results will serve as a test bed to assess the analytical estimates presented in the previous section. Second, the unit cells offer flexibility in the estimation of the homogenized response of the composites since they allow (a) for microstructural variability as well as

(b) for the use of other constitutive descriptions of the constituents of increased complexity.

Previous studies have attempted to model the complex distribution and cross-sectional shape of the muscle fibers using anatomical [48, 49], non-periodic geometrically random [62], or hexagonal unit-cells 
[54]. Complementary to those studies we propose, in this study, a virtual unit cell that has the following properties:

- the unit cell is by construction periodic, [38, 54],

- the cross section of the fibers is polygonal while the fibers themselves are surrounded by finite-thickness ECM zones and distributed randomly allowing to easily reach extremely high volume fractions up to $100 \%$,

- the unit cell contains a sufficiently large number of fibers to ensure isotropy and representativity in the plane normal to the fiber direction,

- the unit cell allows for a good meshing strategy and in general very good mesh quality, which is necessary for the large strain calculations carried out in this study,

- assuming that the composite is invariant along the fiber direction, only one element is used along this direction, thus reducing the cost of the numerical simulations, while allowing for any three dimensional loading condition that produces homogeneous stress and strain fields along the fiber direction of the tissue. This assumption can be easily dropped by assuming a finite fiber length and non-periodic boundary conditions along the fiber direction if required.

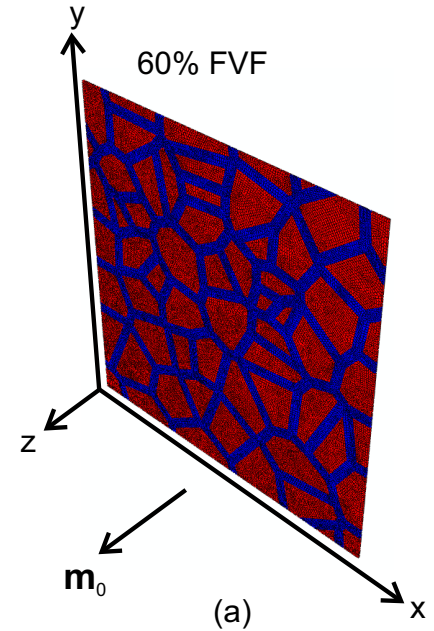

(a)

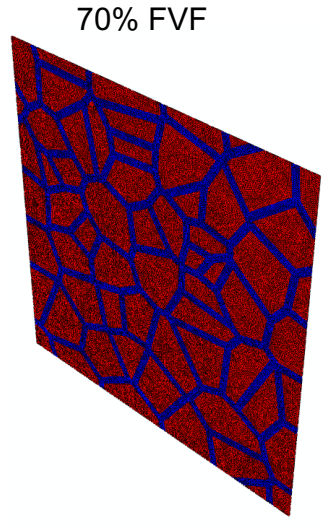

(b)

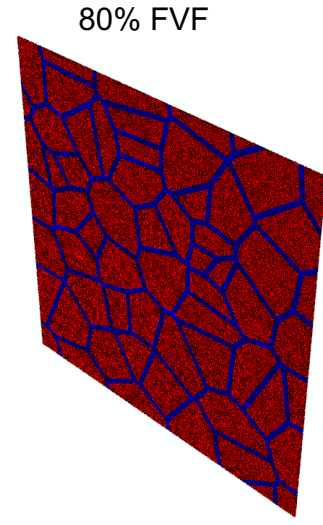

(c)

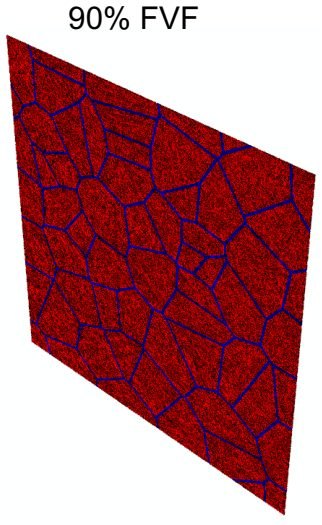

(d)

Figure 4: Periodic Voronoi unit-cell FE meshes with (a) $60 \%$ FVF, (b) $70 \%$ FVF, (c) $80 \%$ FVF, and (d) $90 \%$ FVF. Fibers and ECM are shown in red and blue colour respectively. The initial fiber direction $\mathbf{m}_{0}$ is taken along the global z-axis.

More specifically, Fig. 4 shows representative FE meshes of the unit cell for $c \equiv \mathrm{FVF}=(60 \%, 70 \%, 80 \%, 90 \%)$ used in the calculations. The resulting FVF depends on the ECM thickness, which is taken as constant across the whole volume of each unit cell in the undeformed configuration (Table 1).

Table 1: Thickness of the ECM material for a given FVF. Each Voronoi cell is subjected to an offset that is half the reported thickness. For each value of the thickness, six realizations were generated: the relative standard deviation of the FVF was in all cases of the order of $0.3 \%$.

\begin{tabular}{cc}
\hline \hline FVF & ECM Thickness \\
\hline $60 \%$ & $11.2 \mu \mathrm{m}$ \\
$70 \%$ & $8 \mu \mathrm{m}$ \\
$80 \%$ & $5.2 \mu \mathrm{m}$ \\
$90 \%$ & $2.5 \mu \mathrm{m}$ \\
\hline
\end{tabular}


A total of six realizations for each FVF (see three of them in Fig. 5) were considered sufficient to ensure the statistical representativity of the unit cell due to very low relative standard deviations (RSD) of the FVF (maximum RSD of the order of $0.3 \%$ ) and the average stress for a given value of the strain (maximum RSD of the order of $8 \%$ ). Thus, in the comparisons with the analytical solution (21) that follow in the next section, all presented FE results correspond to the average of six realizations.
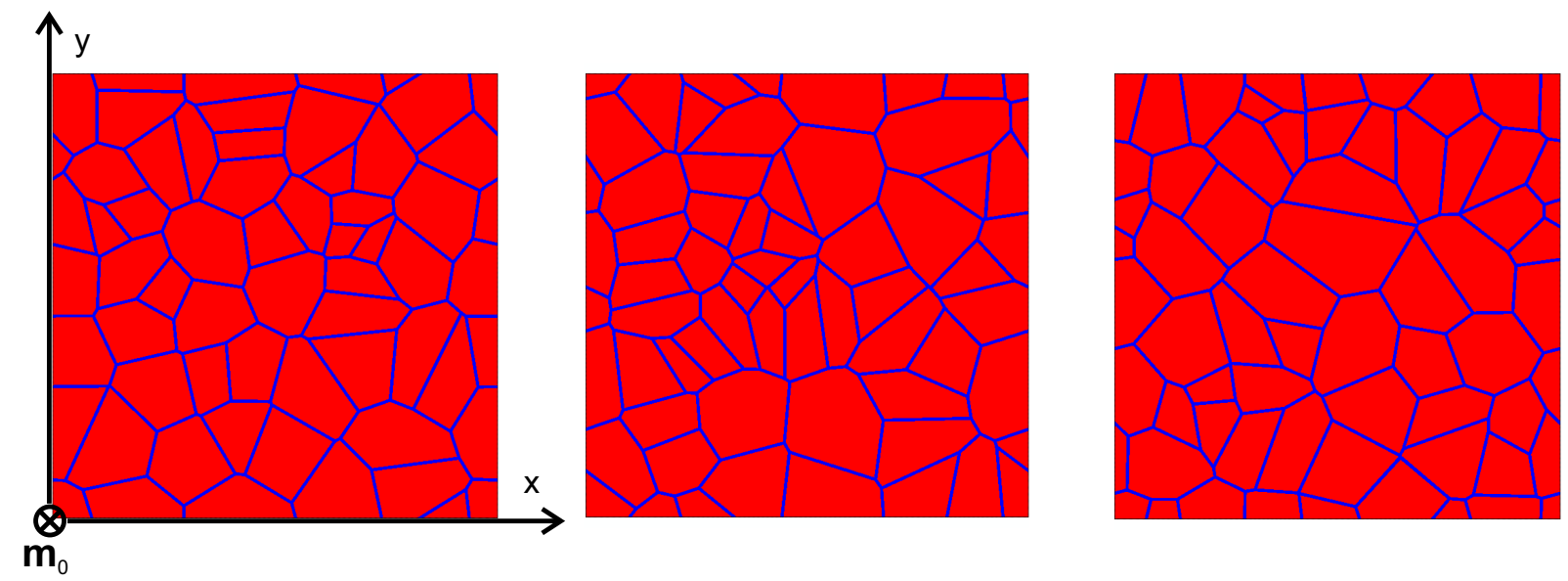

Figure 5: Six realizations for each FVF were analyzed. For illustration purposes we show three out of the six realizations corresponding to $90 \% \mathrm{FVF}$. The unit vector along the muscle fiber direction in the undeformed configuration $\mathbf{m}_{0}$ is normal to the $\mathrm{x}-\mathrm{y}$ plane of the microstructural cross sections.

The finite element calculations are carried out in Abaqus under quasi-static conditions and finite strains. The constitutive models described in Section 2.1 are used to describe the behavior of the constituents. The size of the unit-cell was taken as $350 \mu \mathrm{m} \times 350 \mu \mathrm{m}$. Eight-node hexahedral hybrid elements $(\mathrm{C} 3 \mathrm{D} 8 \mathrm{H}$ in ABAQUS) with a constant pressure stress interpolation are utilized in order to handle efficiently the nearly incompressible behavior of the muscle fibers and ECM. Also, it is assumed that bonding between fibers and ECM is perfect so that a rigid mesh connection between the constituents is applied. A preliminary analysis showed that the behavior of the unit-cell is insensitive to the number of elements used through the out-ofplane direction (fiber direction), thus one element thick meshes are used in the current analysis. In the plane normal to the fiber direction a very fine discretization is used and a minimum number of four elements is inserted through the ECM thickness in all the FVF cases to accurately capture the stress and strain fields developed therein. Mesh sensitivity studies revealed that meshes with approximately $(3,5,10,39) \times 10^{4}$ elements for a unit-cell with $60 \%, 70 \%, 80 \%, 90 \%$ FVF, respectively (such as the meshes shown in Fig. 4) produce converged and accurate results.

\subsection{Generation of unit cells}

Direct observation of histological cross-sections of skeletal muscle (Fig. 1) suggests that the cells of a Voronoi tessellation are a suitable model of muscle fibers. Such geometries are frequently used to perform full-field simulations on polycrystalline microstructures [23]. Special treatement is needed in the present study in order to account for the ECM material that surrounds each individual fiber, as shown in Fig. 6.

First, a random sequential addition algorithm [57] is used to place $N=50$ seeds in the $350 \mu \mathrm{m} \times 350 \mu \mathrm{m}$ unit-cell. Setting the minimum distance between seeds to $10 \mu \mathrm{m}$ ensures that the resulting Voronoi cells are not too small. In order to enforce periodicity, each seed is replicated in the eight neighboring ghost-cells (see Fig. 6a). Owing to the relatively small number of seeds, this fairly simple approach followed for the periodization of the Voronoi tessellations is found to be sufficient [see more efficient approaches in e.g. 64] for the purposes of the present work. 

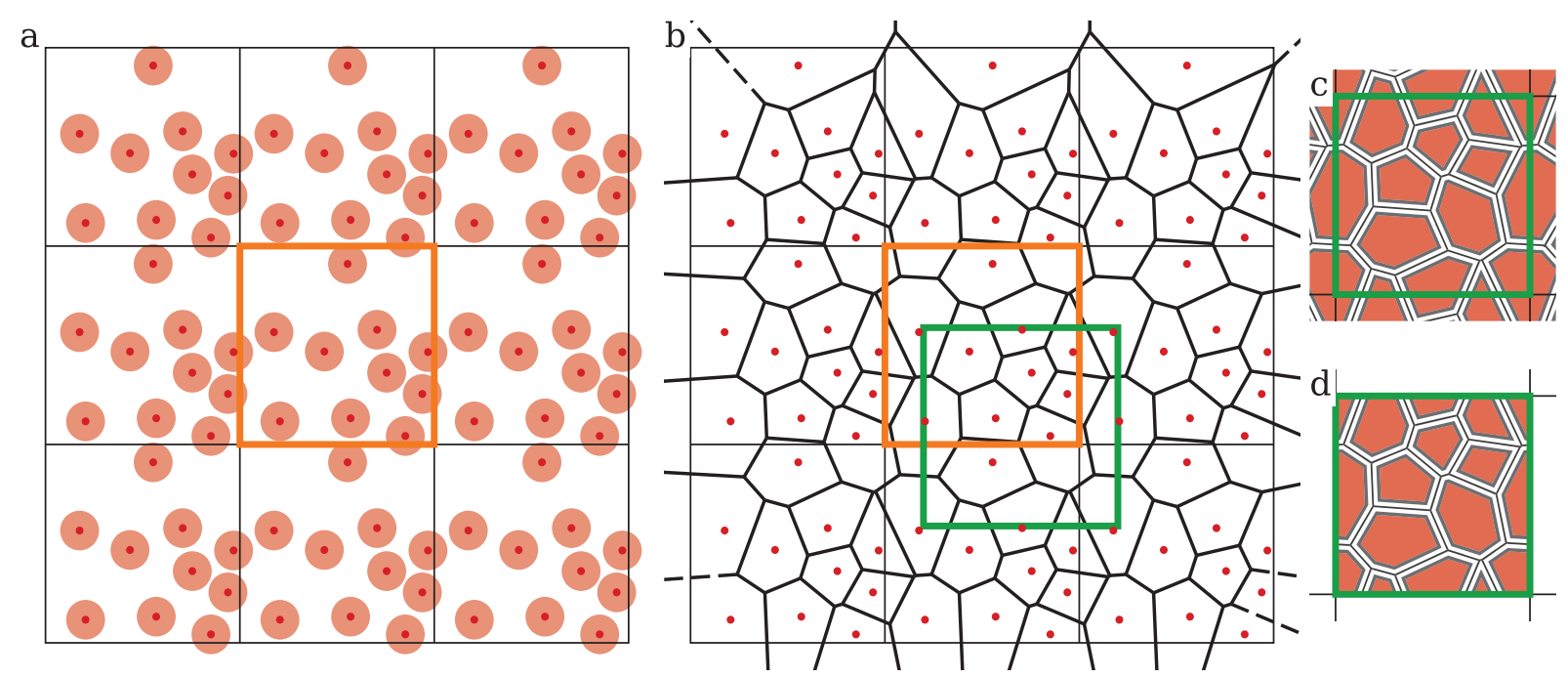

Figure 6: Generation of unit-cells. Seeds are first placed randomly (a), taking into account a minimum center-to-center distance (see shaded disks) through a simple RSA algorithm. The Voronoi tessellation is then generated (b), and the location of the center of the unit-cell is optimized (initial location: orange, optimized location: green). Then, an offset is applied to all Voronoi cells (c). Finally, the microstructure is clipped to the boundaries of the unit-cell (d).

The Qhull library ${ }^{1}$ is then used through its Python/SciPy wrapper ${ }^{2}$ to compute the Voronoi tessellation of the resulting set of $9 N$ seeds (see Fig. 6b). All subsequent geometric operations are carried out through the Python/Shapely library ${ }^{3}$. Vertices that fall too close to the boundary of the unit-cell might affect locally the quality of the generated mesh. To improve on this and taking advantage of the periodicity of the generated microstructure, a translation is subsequently applied to the center of the unit cell in order to maximize the vertex-to-boundary minimum distance (see Fig. 6b).

A constant, negative parallel offset is then applied to each Voronoi cell [63]. This effectively generates constant thickness areas surrounding the Voronoi cells, which represent the ECM material (see Fig. 6c). It should be noted that only the mean value of the FVF is controlled through the value of the offset (see Table 1).

Finally, the various polygonal domains generated are clipped at the boundaries of the unit-cell (see Fig. 6d), and the resulting microstructure is passed to $\mathrm{Gmsh}^{4}$ for mesh generation [12].

\subsection{Periodic boundary conditions}

The application of periodic boundary conditions is necessary in order to be able to extract a homogenized response out of the numerical unit cell. These conditions directly imply that the proposed unit cell can be infinitely repeated in the three directions to provide a representative muscle structure, whereby requiring only the solution of a reduced unit cell to estimate the homogenized response. A detailed description of the implementation of periodic boundary conditions in a finite element setting can be found elsewhere $[34,36,54]$. Here, for completeness, we mention that the periodic boundary conditions are expressed as

$$
\mathbf{u}=(\widehat{\boldsymbol{F}}-\boldsymbol{\delta}) \cdot \mathbf{X}+\mathbf{u}^{*}
$$

where the second-order tensor $\widehat{\boldsymbol{F}}$ denotes the average deformation gradient of the unit-cell which is equal to the applied deformation gradient $[38,21], \mathbf{X}$ denotes the spatial coordinates in the reference configuration, $\boldsymbol{\delta}$ is the second-order identity tensor, and $\mathbf{u}^{*}$ is a periodic displacement field.

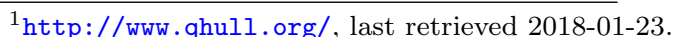

${ }^{2}$ https://docs.scipy.org/doc/scipy/reference/tutorial/spatial.html, last retrieved 2018-01-23.

${ }^{3}$ https://pypi .org/project/Shapely/, last retrieved 2018-01-23.

${ }^{4}$ http://gmsh.info/, last retrieved 2018-01-23.
} 
The volume average true stress tensor in the unit-cell $\widehat{\boldsymbol{\sigma}}$ is calculated as $\widehat{\boldsymbol{\sigma}}=\sum_{i} \boldsymbol{\sigma}_{i} V_{i} / \sum_{i} V_{i}$ where $\sigma_{i}$ and $V_{i}$ are the true stress tensor evaluated at the centroid and the volume of the $i$-th finite element respectively. The volume average nominal (1st Piola-Kirchhoff) stress tensor $\widehat{\boldsymbol{t}}$ is then computed by the relation $\widehat{\boldsymbol{t}}=\widehat{J} \widehat{\boldsymbol{F}}^{-1} \cdot \widehat{\boldsymbol{\sigma}}$. Here, $\widehat{J}=\operatorname{det} \widehat{\boldsymbol{F}}$, where $\widehat{\boldsymbol{F}}$ corresponds to the average deformation gradient in the unit cell and has been defined via the periodic boundary conditions in Eq. (33). All loadings are characterized by the macroscopic applied average deformation gradient $\widehat{\boldsymbol{F}}$.

For the problem of uniaxial tension in direction $i$ the deformation gradient is of the form

$$
\widehat{\boldsymbol{F}}=\widehat{\lambda} \mathbf{e}_{i} \mathbf{e}_{i}+\widehat{\lambda}_{t}\left(\mathbf{e}_{j} \mathbf{e}_{j}+\mathbf{e}_{k} \mathbf{e}_{k}\right), \quad \forall i, j, k=1,2,3 \quad \text { and } \quad i \neq j \neq k
$$

where $\left(\hat{\lambda}, \hat{\lambda}_{t}\right)$ are the average axial and transverse stretch ratios and $\left(\mathbf{e}_{i}, \mathbf{e}_{j}, \mathbf{e}_{k}\right)$ are the base vectors along the coordinate axes shown in Fig. 4 . It is noted that $\widehat{\lambda}_{t}$ is not directly applied but is a result of imposing a zero average transverse true stress. This is simply achieved by letting the lateral master nodes free (see Mbiakop et al. [36] for more details on applying a fixed stress triaxiality load).

For the problem of simple shear, we completely set the deformation gradient to be of the form

$$
\widehat{\boldsymbol{F}}=\boldsymbol{\delta}+\widehat{\gamma} \mathbf{e}_{i} \mathbf{e}_{j}, \quad \forall i, j=1,2,3 \quad \text { and } \quad i \neq j,
$$

where $\widehat{\gamma}$ is the average amount of shear on the $i-j$ plane.

\section{Results and discussion}

In this section, first we make use of experimental results available in the literature to identify the analytical model parameters and then we compare the predictions of the analytical homogenization model for the skeletal muscle developed in section 2 with the FE results of the numerical homogenization model discussed in section 3 for the full range of 3D loadings. In particular, we examine the influence of FVF $(c)$ and shear moduli contrast on the effective muscle response in the muscle fiber direction. Next, we examine whether the analytical model is able to capture the muscle response under normal (in-plane) and shear loadings.

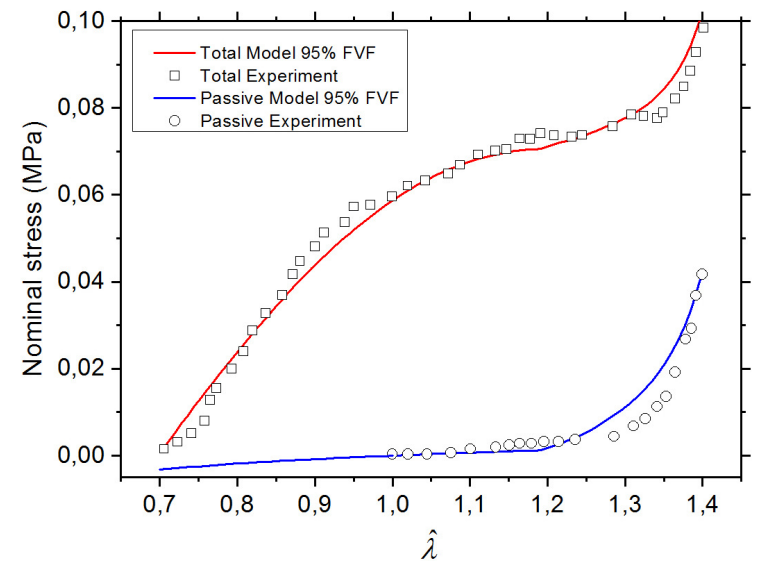

(a)

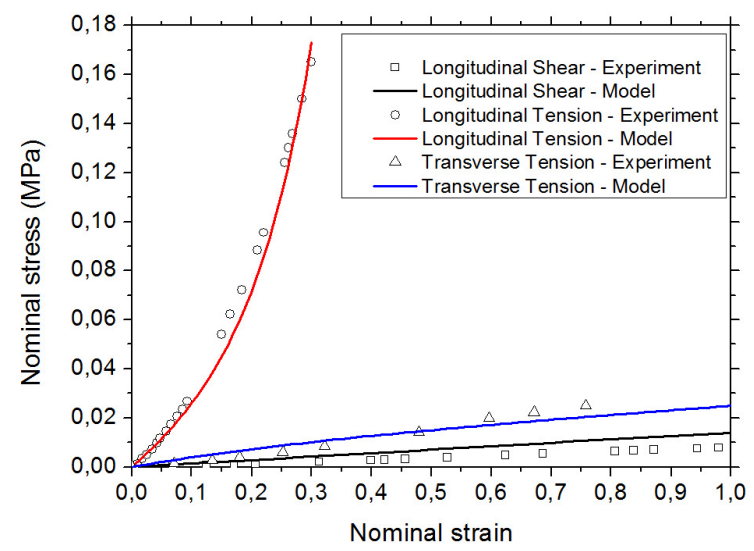

(b)

Figure 7: Analytical homogenization model comparison with the experimental data of (a) Hawkins and Bey [13] under uniaxial loading in the muscle fiber direction, and (b) Morrow et al. [40] under longitudinal tension, transverse tension, and longitudinal shear. 


\subsection{Identification of the model parameters}

In order to identify the model parameters, the analytical homogenization model is compared to the experimental data of Hawkins and Bey [13], who measured the passive and active force-length response of a normal rat tibialis anterior muscle. During those active force-length tests the muscle was stimulated isometrically (constant muscle length), thus the normalized force-velocity function that enters in Eq. (8) is set to unity $\left(f_{r}\left(\dot{\lambda}_{m}\right)=1\right)$ implying no dependency of the velocity (stretch rate) of contraction on the active stress. Due to the small pennation angle of the rat's tibialis anterior muscle fibers we assume that the experimental loading direction coincides with the muscle fiber direction [10]. The value for the fiber volume fraction $c(\equiv \mathrm{FVF})$ that appears in Eq. (23) is set to $c=95 \%$ according to Lieber et al. [28] data for normal muscles. The value for $\sigma_{\max }$ that enters Eq. (8), as well as the values for $\lambda_{m}^{\text {opt }}$ and $\lambda_{m}^{\min }$ that enter the active stress-stretch function $f_{e}$ in Eq. (10) are directly determined from the stress-stretch curve of Hawkins and Bey [13] as $\sigma_{\max }=73 \mathrm{kPa}, \lambda_{m}^{\text {opt }}=1.192$, and $\lambda_{m}^{\min }=0.682$. Furthermore, the muscle fiber's passive elastic modulus $E_{p}$ that enters the passive stress-strain function $f_{p}^{(1)}$ is set to $E_{p}=63 \mathrm{kPa}$ based on the single fiber experimental results of Thacker et al. [55] on rat tibialis anterior muscles. Also, we assume fully active muscle fibers $\left(f_{a}=1\right)$ when the activity of the muscle is taken into account and $f_{a}=0$ when muscle exhibits only passive behavior. The values of the rest model parameters $A 1, A 2$ and $\theta_{0}$ that enter the function $f_{p}^{(2)}$ in Eq. (16) and the shear moduli of the muscle's constituents $G^{(1)}, G^{(2)}$ are assigned as $G^{(1)}=2.5 \mathrm{kPa}, G^{(2)}=1 \mathrm{kPa}, A 1=53 \mathrm{kPa}, A 2=110$, and $\theta_{0}=59^{\circ}$ in order to achieve a good agreement with the experimental data as shown in Fig. 7a, taking into account that the shear moduli of the muscle's constituents are in the order of few $\mathrm{kPa}$ [60]. Finally, we assign the corresponding bulk moduli to be 10,000 times the shear moduli in order to account for the incompressible behavior of the constituents $\left(K^{(r)}=10^{4} G^{(r)}, r=1,2\right)$.

In addition, the analytical homogenization model is compared to the experimental measurements of Morrow et al. [40], who tested extensor digitorum longus rabbit muscles under tension in the muscle fiber direction (longitudinal tension), tension in a direction transverse to the muscle fiber direction, and longitudinal shear along the muscle fiber direction. By adjusting the model parameters to be $G^{(1)}=15 \mathrm{kPa}$, $G^{(2)}=5 \mathrm{kPa}, E_{p}=100 \mathrm{kPa}, \lambda_{m}^{\text {opt }}=1, A 1=170 \mathrm{kPa}, A 2=12, \theta_{0}=25^{\circ}$, and keeping $c=0.95$, Fig. $7 \mathrm{~b}$ shows that the analytical model is able to probe accurately the experimentally measured muscle response under all the three distinct loading modes.

\subsection{Assessment of the analytical homogenization model by comparison with the numerical model}

In this section we assess the accuracy of the analytical homogenization muscle model for different volume fractions of the constituents by comparing its predictions to the results of the numerical homogenization model. Since experimental measurements for the shear moduli of the constituents themselves do not currently exist in the literature we examine skeletal muscle response over a wide range of shear modulus contrast between muscle fiber and ECM $G^{(1)} / G^{(2)}=5,10,20,50$, with $G^{(2)}=1 \mathrm{kPa}$. The remaining values of the model parameters are those that match the experimental data of Hawkins and Bey [13] as reported in the previous section. It is also important to note at this point that in the current literature, there exist - to the best of our knowledge - no experiments that investigate the effect of muscle fiber volume fraction upon the overall muscle response. As a consequence, in the following sections, we assess the accuracy of the analytical homogenization model by comparison with the corresponding numerical model. That is a highly non trivial assessment since the nonlinearities exhibited by the muscle phases are strong and the volume fractions of the fibers very high.

\subsubsection{Fiber direction response}

For loadings in the muscle fiber direction the deformation gradient field that is developed in the RVE is uniform and as a consequence the developed stress fields are constant-per-phase. Therefore, the proposed analytical homogenization model reduces to the homogenization model of the Voigt type as presented in Spyrou et al. [54], and thus the two models produce the same exact estimates for that type of loading conditions. It is noted here again that for incompressible transversely isotropic skeletal muscles the Voigt estimate is exact for axisymmetric loading conditions aligned with the muscle fiber direction, i.e. the 


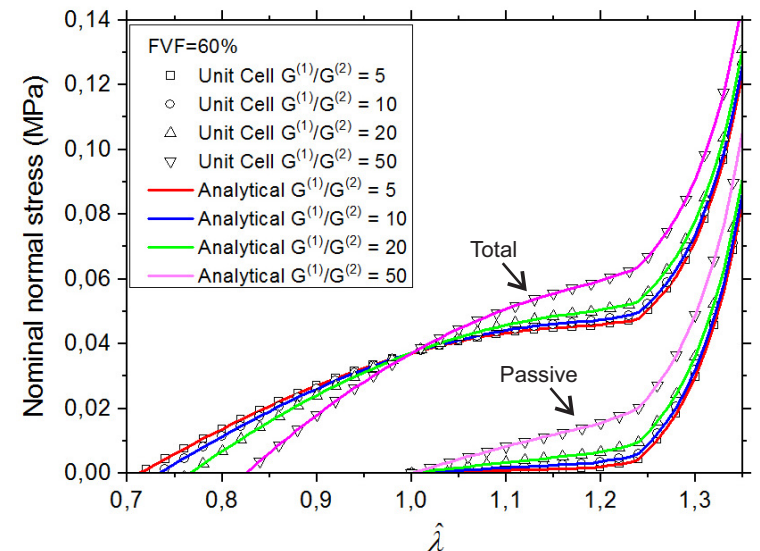

(a)

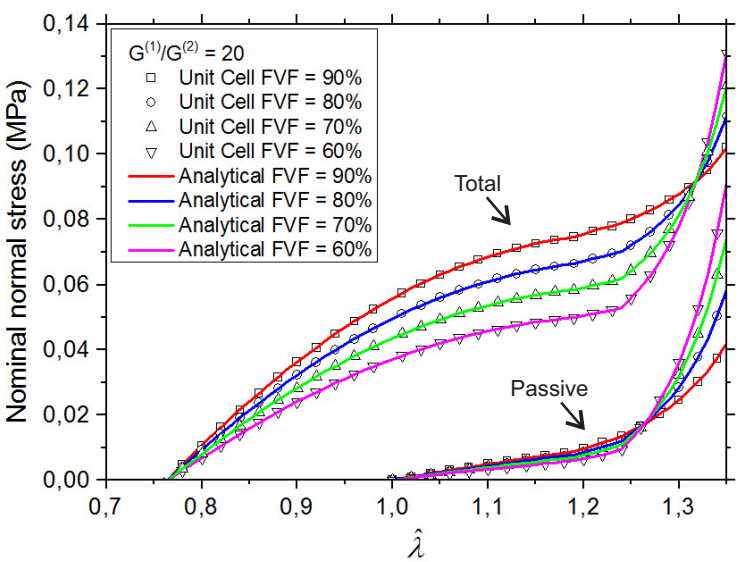

(c)

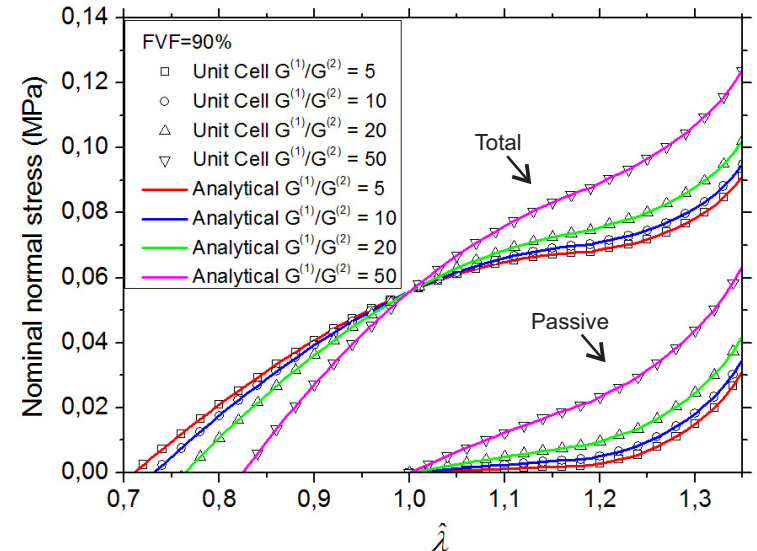

(b)

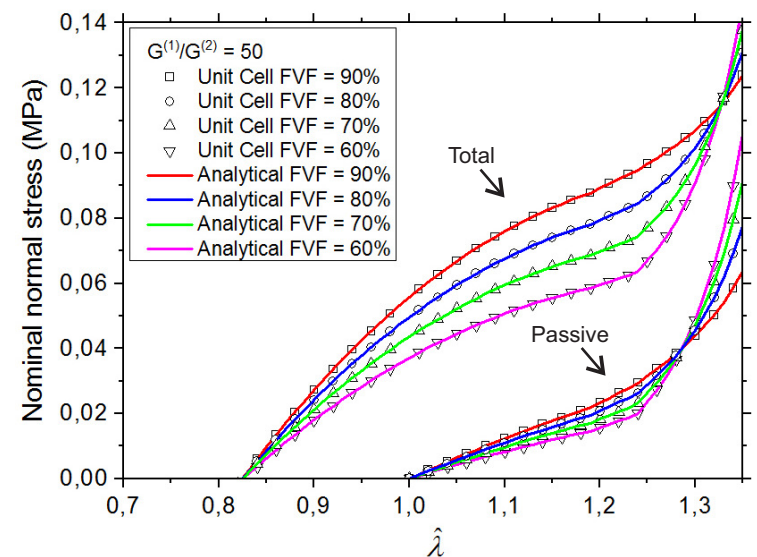

(d)

Figure 8: Muscle response under loadings in the muscle fiber direction. Analytical model predictions compared to FE unit-cell simulations for (a) FVF $=60 \%$ and various shear moduli contrasts $G^{(1)} / G^{(2)}$, (b) FVF $=90 \%$ and various shear moduli contrasts $G^{(1)} / G^{(2)}$, (c) $G^{(1)} / G^{(2)}=20$ and various FVF, and (d) $G^{(1)} / G^{(2)}=50$ and various FVF. The shear modulus of ECM is taken as $G^{(2)}=1 \mathrm{kPa}$. The same agreement between analytical and numerical results is also achieved for the intermediate $\mathrm{FVFs}=70 \%, 80 \%$.

symmetry axis of transverse isotropy in skeletal muscles. For the sake of completeness, in Fig. 8 we present the comparison between the analytical model predictions and the FE unit-cell calculations, which shows excellent agreement. In all cases considered the predicted total muscle response is consistent with the experimentally observed trend shown in Fig. 7a.

As already shown in Spyrou et al. [54] for that particular loading case, at large enough tensile stretching in the fiber direction, the muscle response may become much stiffer with the increase of the ECM volume fraction, whereby a cross-over of the total response occurs (Figs. 8c and 8d). In particular, at low stretch levels, the response is dominated by the isotropic behavior of the constituents, thus the higher FVF leads to higher stresses when $G^{(1)} / G^{(2)}>0$. At higher stretches $(\sim>30 \%)$ this response is reversed, i.e. the anisotropic (fibrous) part of the constituents dominates the whole response. Therefore, by increasing the volume fraction of ECM and stretching levels, more and more collagen fibers become aligned with the muscle fiber direction, thus causing the exponential increase of muscle's stiffness due to their high nonlinear behavior. Interestingly, such a cross-over has been previously observed experimentally in Smith et al. [50], where the passive response of fiber bundles including ECM was compared between normal and spastic muscles; the spastic muscles had a significantly higher collagen content than the normal muscles. The increased muscle stiffness of spastic muscles when stretched in the fiber direction due to their higher content in ECM compared 
with the healthy subjects was also observed in that study. In addition, our findings probe successfully the experimental results of Meyer and Lieber [37], who revealed the primary contribution of ECM on muscle's stiffness and highly nonlinear response under tensile loading in the muscle fiber direction.

On the other hand, only a large enough shear moduli contrast between muscle fibers and ECM (e.g. $\left.G^{(1)} / G^{(2)}>20\right)$ can have a significant effect on the total anisotropic tissue response, as shown in Figures $8 \mathrm{a}$ and $8 \mathrm{~b}$. For example, the minimum fiber stretch at which muscle begins to develop positive total stresses is increased from $\sim 0.72$ to $\sim 0.83$ when the contrast of the shear moduli ratio is increased from $G^{(1)} / G^{(2)}=20$ to $G^{(1)} / G^{(2)}=50$. However, for $\widehat{\lambda}<1$ the total response is dominated by the active behavior of the muscle fibers and directly attributed to the muscle fiber's normalized active stretch-dependent behavior described by the $f_{e}$ function in Eq. (10).
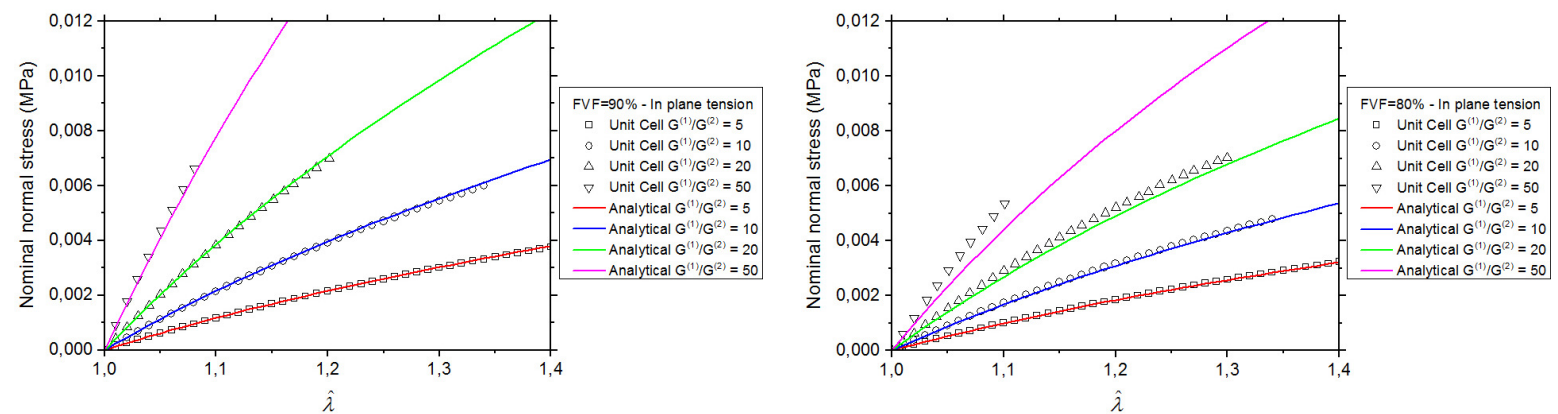

(a)

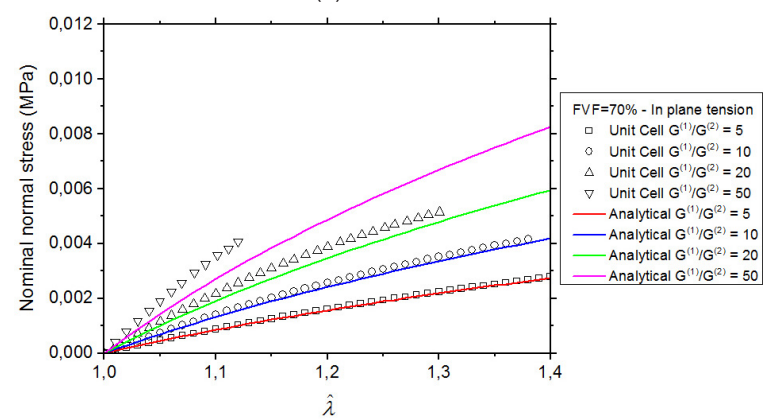

(b)

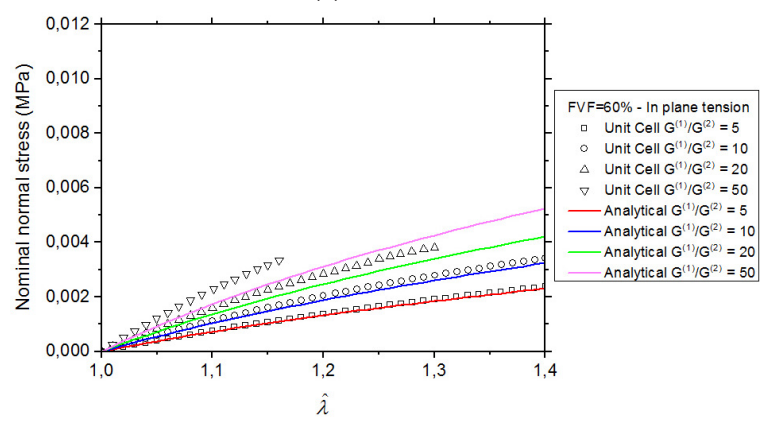

(d)

Figure 9: In-plane tensile behavior for various shear moduli contrasts when (a) $\mathrm{FVF}=90 \%,(\mathrm{~b}) \mathrm{FVF}=80 \%,(\mathrm{c}) \mathrm{FVF}=70 \%$, and $(\mathrm{d}) \mathrm{FVF}=60 \%$.

\subsubsection{In-plane normal response}

The muscle's in-plane normal response is associated with the combined isotropic (Neo-Hookean) behavior of its constituents. Due to the isotropy in the plane normal to the fiber direction, the material response is the same under either $\widehat{F}_{x x}$ or $\widehat{F}_{y y}$ loading (see Figures 4 and 5 for the loading axes). Fig. 9 shows a very good agreement between the analytical and numerical results for most of the cases analysed. The deviation between the aforementioned results increases with decreasing FVF and increasing $G^{(1)} / G^{(2)}$ contrast. The maximum difference between the average stresses for a given value of the stretch is about $20 \%$ and corresponds to a material with $\mathrm{FVF}=60 \%$ and $G^{(1)} / G^{(2)}=50$ when subjected to about $15 \%$ stretch. As can be observed in Fig. 9, due to problems with the numerical convergence, the numerical calculations are stopped at lower overall stretch levels as the FVF and $G^{(1)} / G^{(2)}$ are increased. This typically occurs whenever the elements in the ECM regions become exceedingly distorted because of the high local strains developed.

Fig. 10 presents two examples of large local deformations in ECM regions for the case of FVF $=90 \%$ and $G^{(1)} / G^{(2)}=50$ under in-plane tension (Fig. 10a) and in-plane shear (Fig. 10b). Fig. 10a shows contour plots of the maximum principal logarithmic strain at an overall stretch level of $\widehat{\lambda}=0.1$ whereas Fig. 10b shows the same contour plots at an overall shear strain level of $\widehat{\gamma}=0.15$. In both cases the deformation 


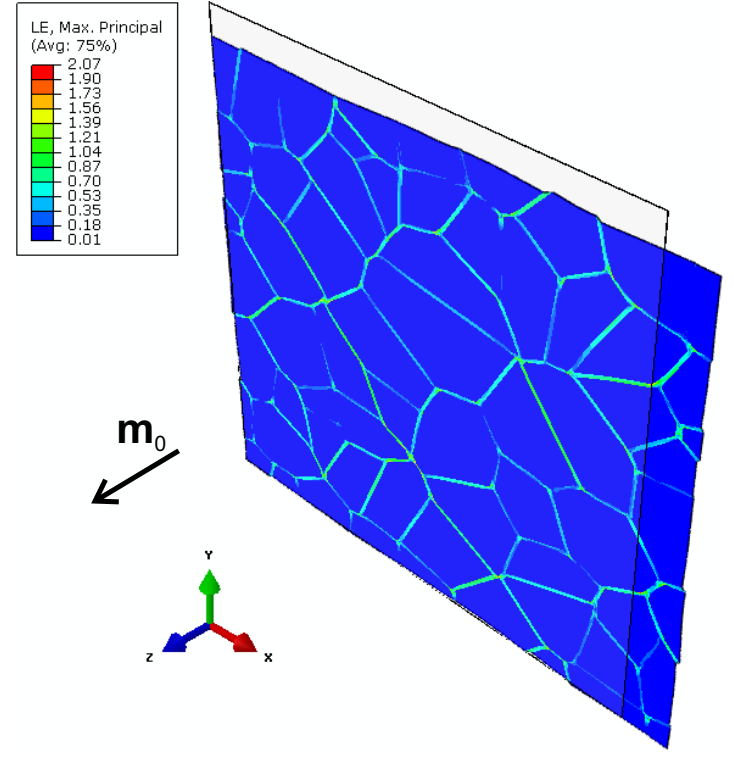

(a)

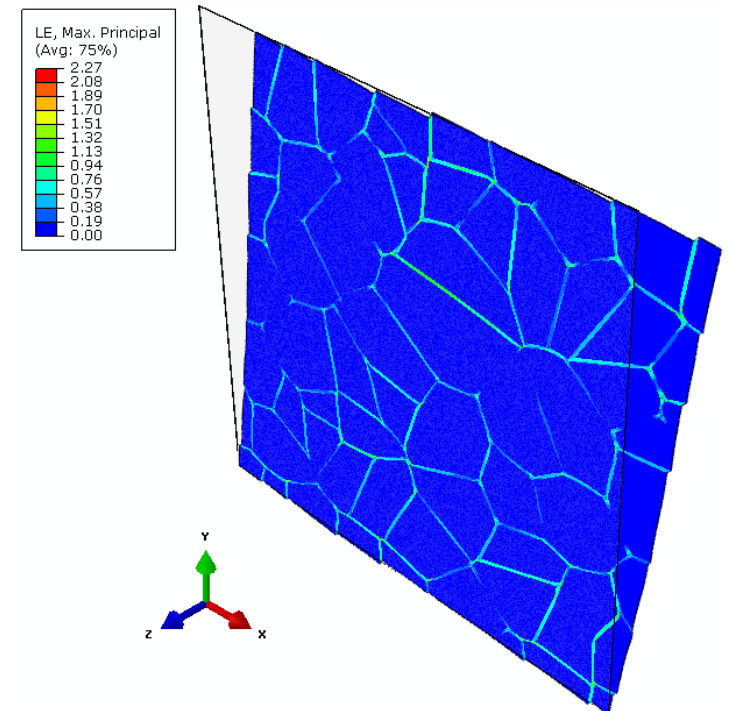

(b)

Figure 10: Contour plots of the maximum principal logarithmic strain for a realization with FVF $=90 \%$ and $G^{(1)} / G^{(2)}=50$ subjected to an overall (a) in-plane tensile stretch of $\widehat{\lambda}=0.1$, and (b) in-plane shear strain of $\widehat{\gamma}=0.15$. In both cases the initial undeformed configuration is also depicted for comparison purposes. The initial fiber direction $\mathbf{m}_{0}$ is taken along the global z-axis.

contours are seen to be highly heterogeneous in the ECM with principal logarithmic strains as large as 2.07 in the case of in-plane tension and 2.27 in the case of in-plane shear.

\subsubsection{Shear response}

During physical function muscle may be deformed under three distinct shearing modes: a) shear in the direction of the fibers (out-of-plane longitudinal shear) by applying $\widehat{F}_{z x}$ or $\widehat{F}_{z y}$, b) shear in the planes normal to the fibers (in-plane shear) by applying $\widehat{F}_{x y}$ or $\widehat{F}_{y x}$, and c) shear across the fibers (out-of-plane perpendicular shear) by applying $\widehat{F}_{x z}$ or $\widehat{F}_{y z}$ (Fig. 11). It is noted that analytical and FE unit-cell calculations result in the same muscle response under either $\widehat{F}_{x z}$ or $\widehat{F}_{y z}$ loading, under either $\widehat{F}_{x y}$ or $\widehat{F}_{y x}$ loading, and under either $\widehat{F}_{z x}$ or $\widehat{F}_{z y}$ loading.

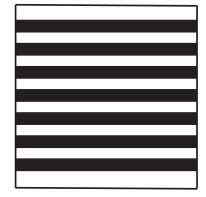

undeformed

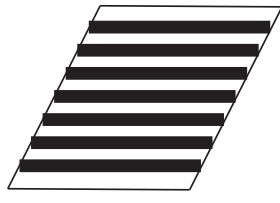

deformed

(a)

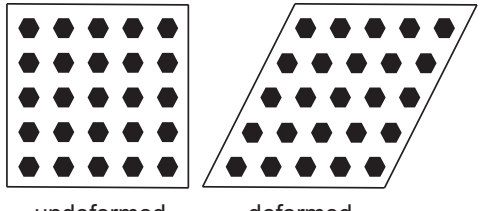

(b)

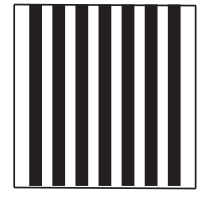

undeformed

(c)

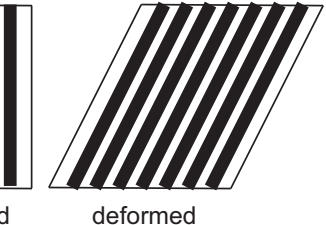

Figure 11: Schematic representation of (a) out-of-plane longitudinal shear, (b) in-plane shear, (c) out-of-plane perpendicular shear. In (a) and (c) the dark lines represent the fibers whereas in (b) the dark polygons denote the cross sections of fibers out of the plane of the page $([9])$.

Fig. 12 shows an excellent agreement between the analytical estimates and the numerical calculations of muscle response under perpendicular shear. The results show that muscle fibers control the overall muscle response when shear strains are maintained under a certain threshold $(\simeq 0.7-0.8$ in the present case $)$; this threshold depends on the orientation of the ECMs collagen fibers with respect to the muscle fiber direction. 


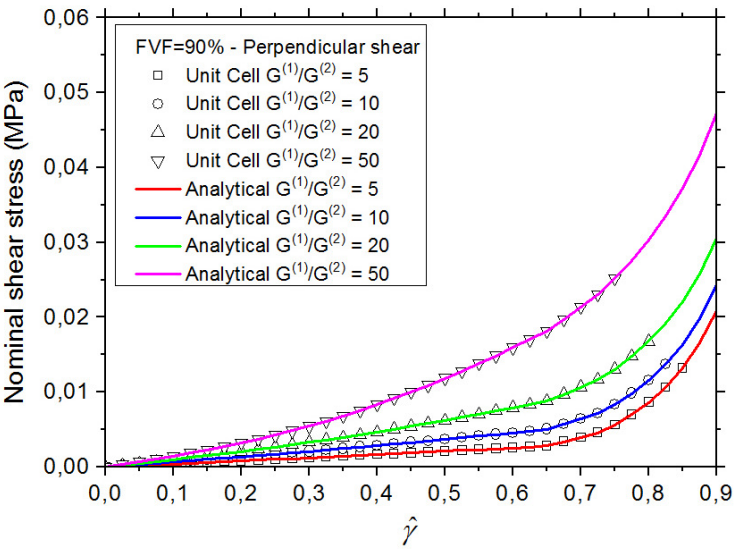

(a)

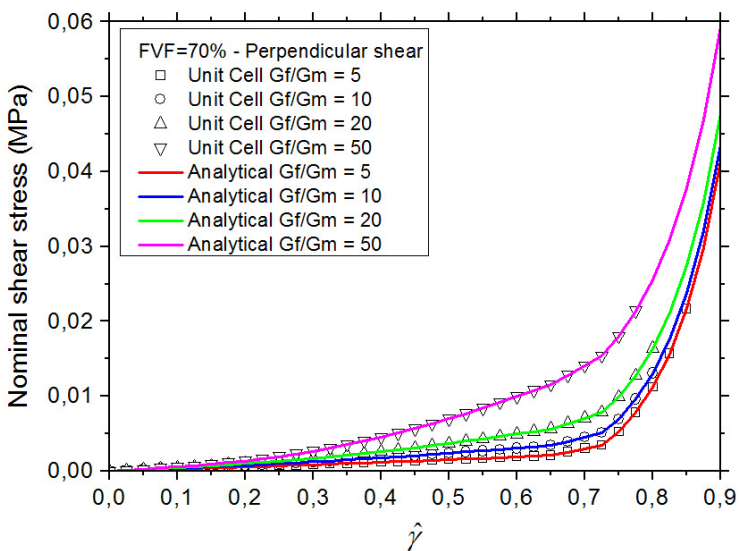

(c)

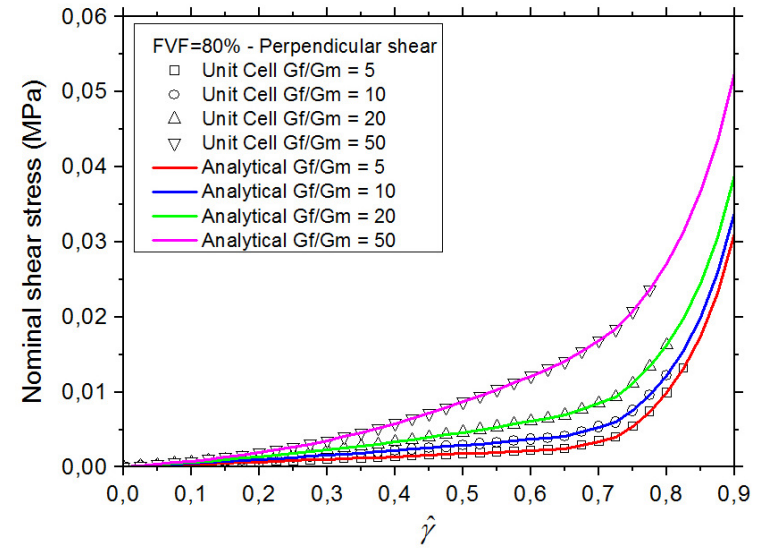

(b)

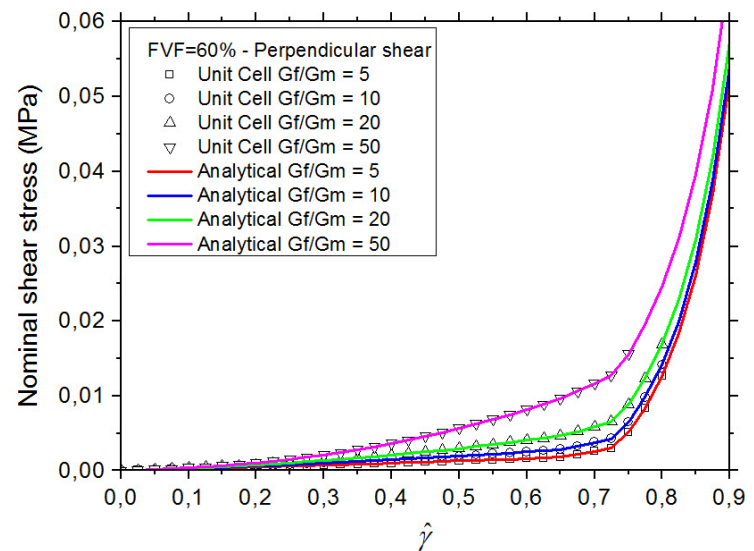

(d)

Figure 12: Out-of-plane perpendicular shear behavior for various shear moduli contrasts when (a) FVF = 90\%, (b) FVF $=80 \%,(\mathrm{c}) \mathrm{FVF}=70 \%$, and (d) $\mathrm{FVF}=60 \%$.

Above this threshold the ECMs collagen fibers are properly oriented in order to become stretched enough and contribute significantly to the overall muscle response. With further increase of perpendicular shearing the effective response becomes increasingly stiffer due to the domination of the ECMs high nonlinear tensile behavior in the fiber direction as explained in Section 4.2.

Note that, for transversely isotropic materials, the out-of-plane perpendicular shear is the only mode of simple shear which causes stretching in the direction of transverse isotropy (fiber stretching), thus resulting in the negative or reverse Poynting effect as described in Destrade et al. [9]. As a consequence of this simple shear deformation mode the sheared faces of the material tend to meet each other causing the development of a tensile normal stress in the direction perpendicular to the applied shear deformation (see also the relevant discussion in Spyrou et al. [54]).

A very good agreement is also achieved under in-plane shear (Fig. 13) and out-of-plane longitudinal shear (Fig. 14) loadings for most of the cases analyzed. As in the in-plane normal response, the deviation between the analytical and numerical results increases with decreasing FVF and increasing contrast $G^{(1)} / G^{(2)}$. The maximum difference between the average stresses for a given value of the stretch is about $15 \%$ and corresponds to in-plane shear muscle response with $\mathrm{FVF}=60 \%$ and $G^{(1)} / G^{(2)}=50$. 

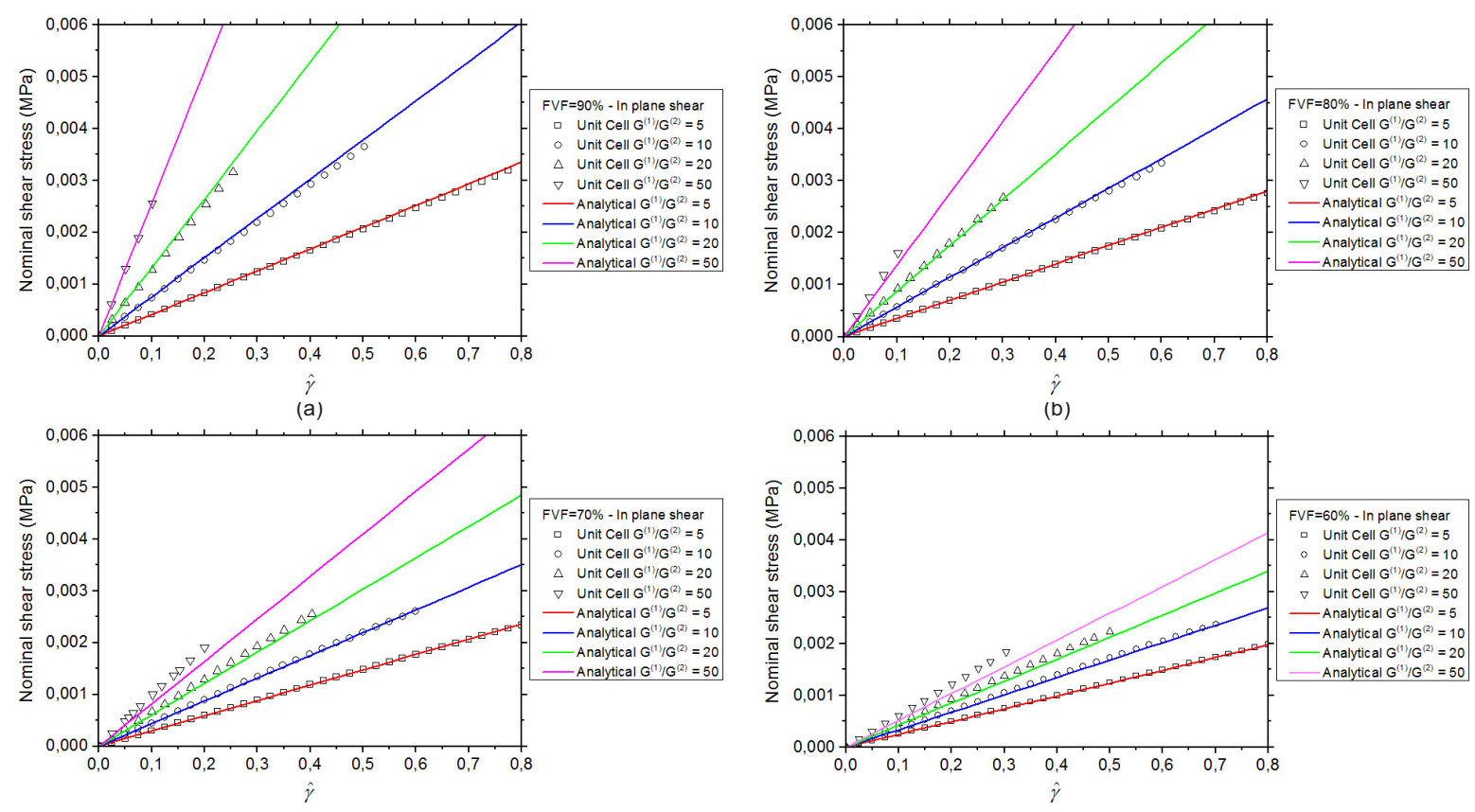

(c)

(d)

Figure 13: In-plane shear behavior for various shear moduli contrasts when (a) FVF $=90 \%$, (b) FVF $=80 \%,(c) \mathrm{FVF}=70 \%$, and (d) FVF $=60 \%$.

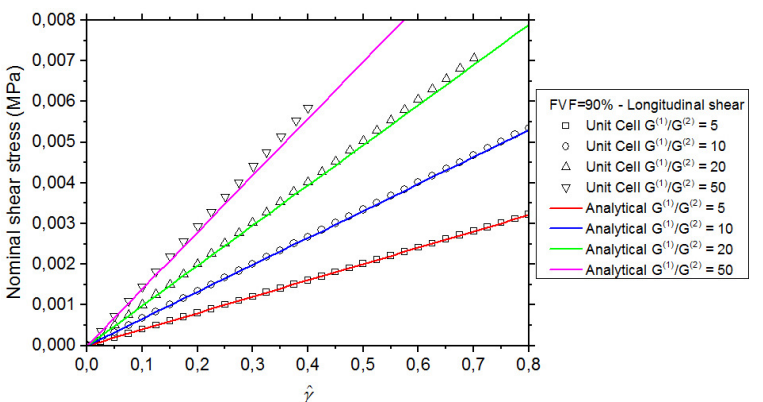

(a)

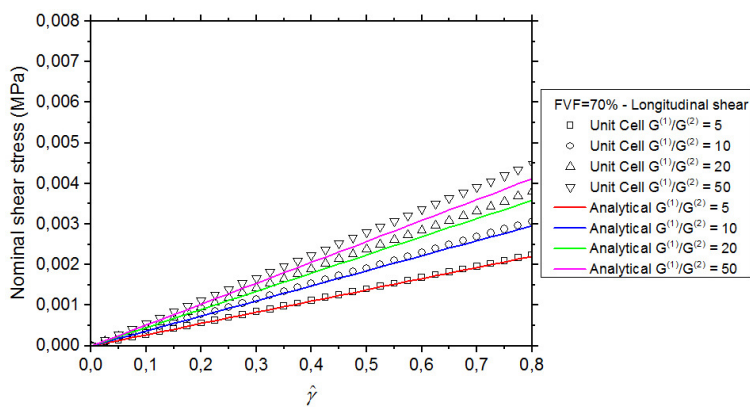

(c)

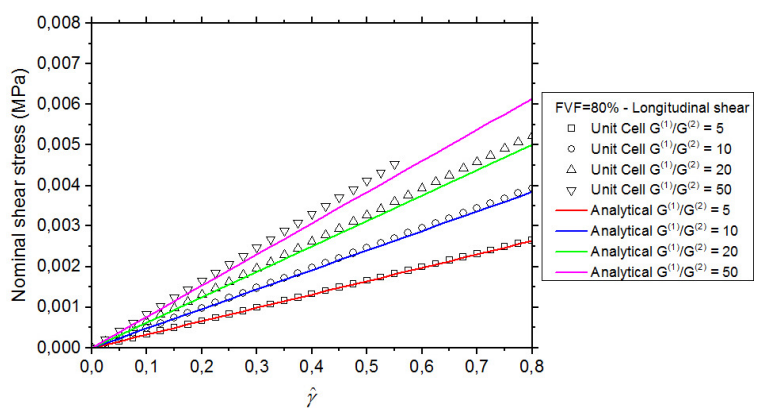

(b)

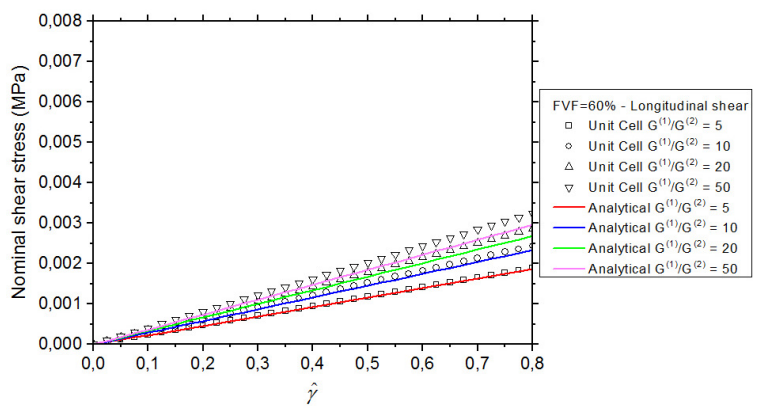

(d)

Figure 14: Out-of-plane longitudinal shear behavior for various shear moduli contrasts when (a) FVF $=90 \%,(b) \mathrm{FVF}=80 \%$, (c) $\mathrm{FVF}=70 \%$, and (d) $\mathrm{FVF}=60 \%$. 


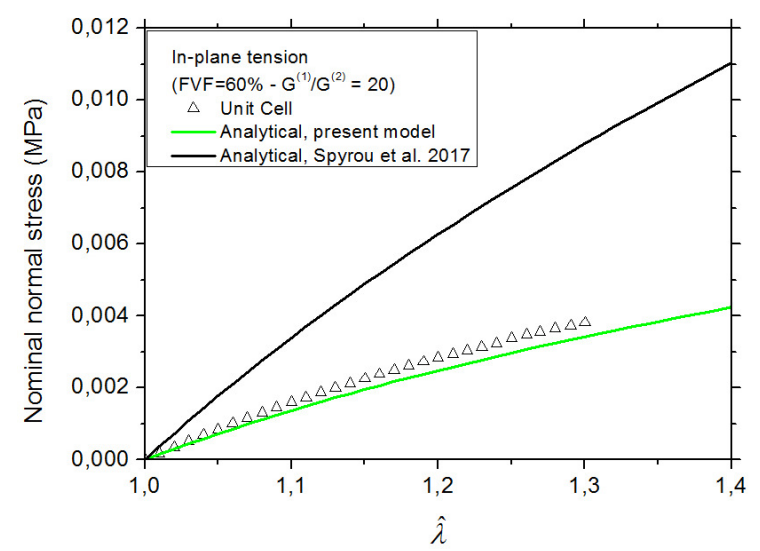

(a)

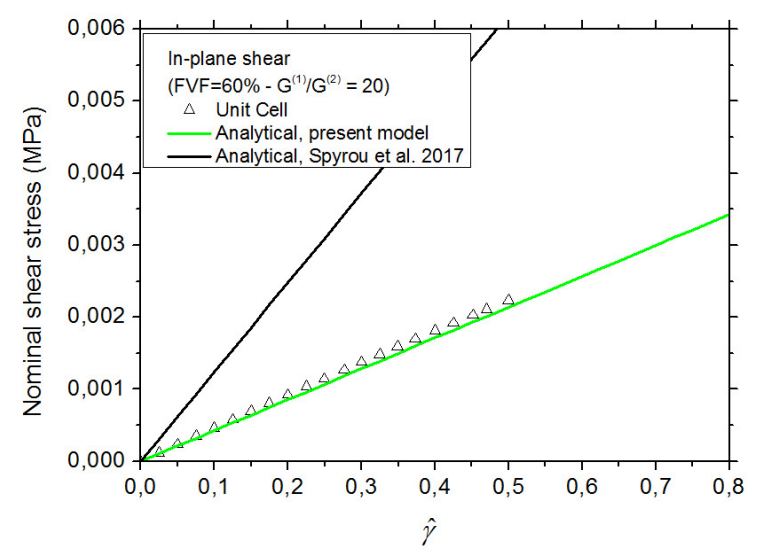

(c)

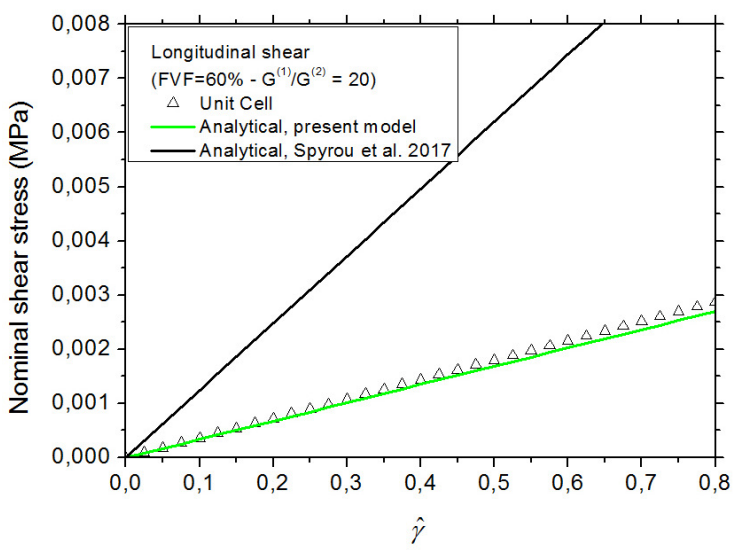

(b)

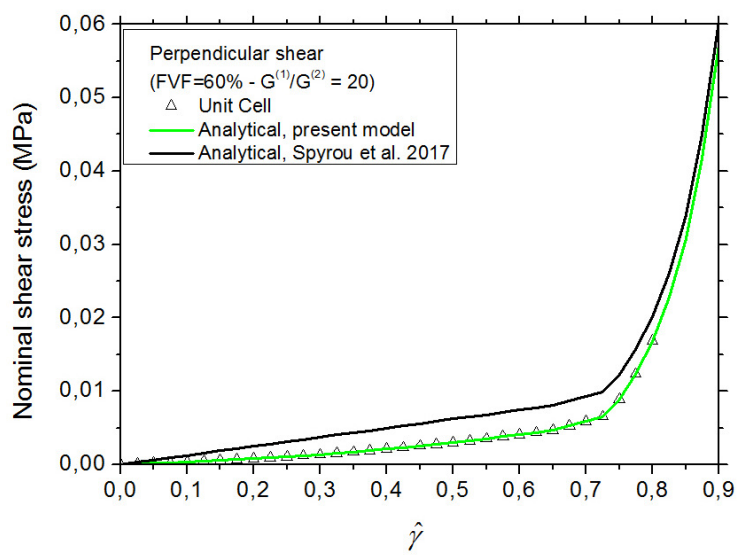

(d)

Figure 15: Muscle response as predicted by the present model and the model of Spyrou et al. [54] for FVF $=60 \%$ and $G^{(1)} / G^{(2)}=20$ under (a) in-plane tension, (b) longitudinal shear, (c) in-plane shear, and (d) perpendicular shear.

\subsection{Comparison of the present analytical homogenization model with the analytical model of Spyrou et al. [54]}

In this section we focus on the differences of the proposed analytical model with the previous homogenization model of the Voigt type of Spyrou et al. [54]. As already discussed in Section 4.2.1 the two models produce the same estimates under loadings in the muscle fiber direction. However, this is not the case for shear or in-plane normal loading conditions. Under these loadings the model of Spyrou et al. [54] produces inaccurate estimates, especially with the increase of the heterogeneity contrast of the constituents and the decrease of the FVF. By contrast, the present model shows remarkable predictive qualities, as shown in Figures 15 and 16. The closest agreement between the two models is observed for the perpendicular shear loading case because it involves fiber stretching which seems to have a major contribution on the overall behavior of the tissue.

\subsection{Mechanical response of $3 D$ muscle macro-structures}

In the previous sections the analytical model for skeletal muscle has been assessed through experimental and extensive FE simulations for various loading conditions and several fiber volume fractions (FVF). This section presents an application of the analytical homogenization muscle model on idealized geometries of fusiform and pennate muscles in order to examine qualitatively their mechanical response when subjected to microstructural changes. The objective of this section is to show that the analytical multiscale muscle 


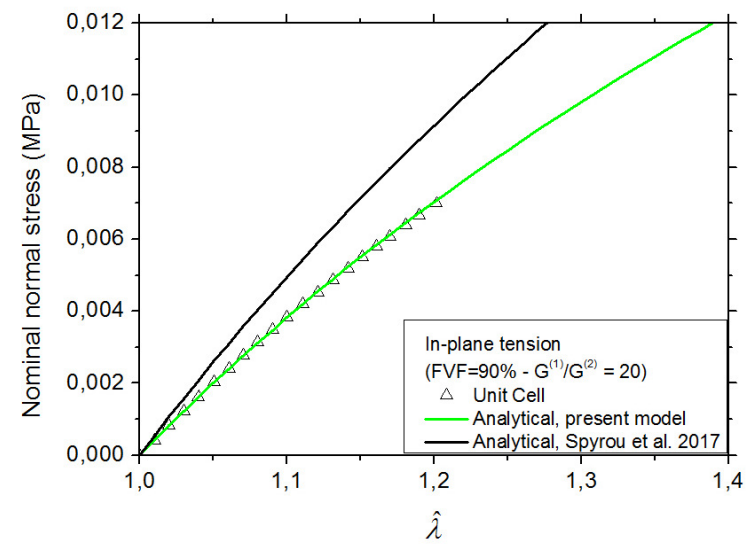

(a)

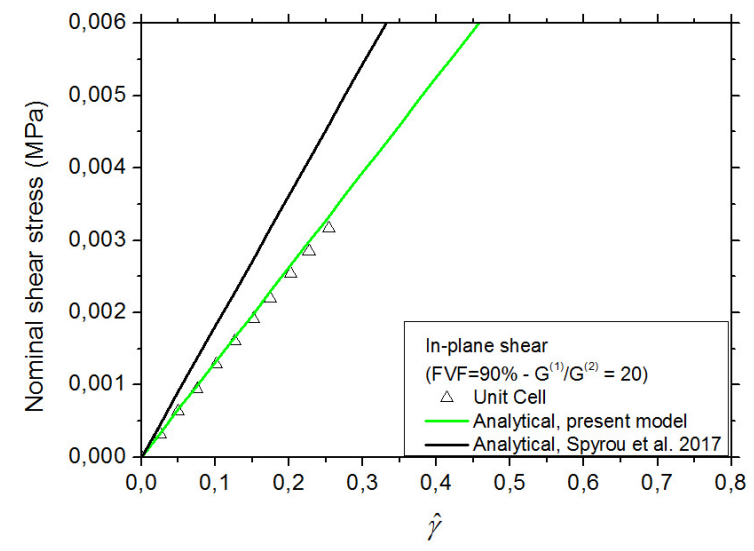

(c)

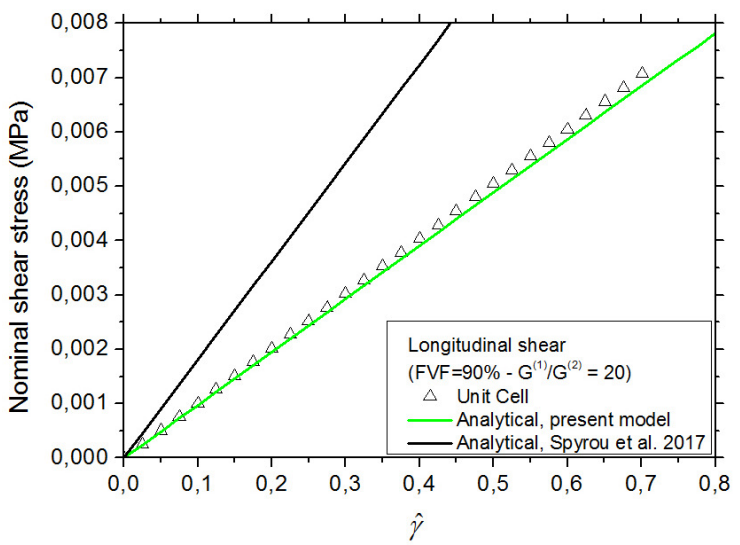

(b)

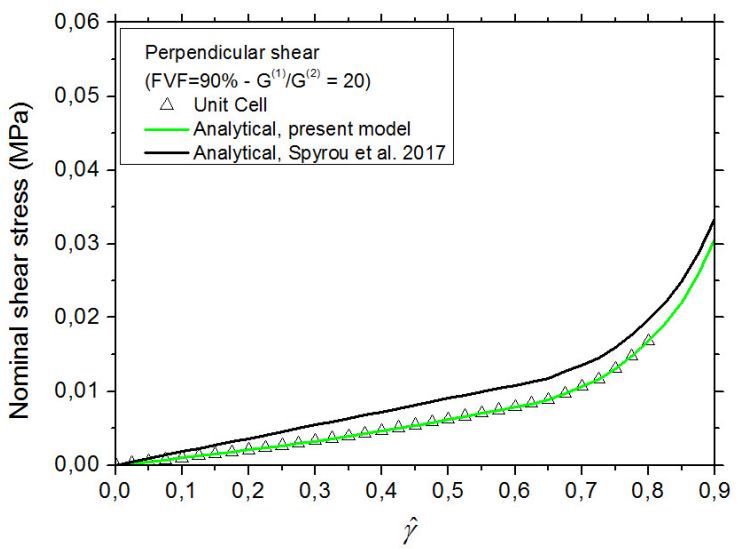

(d)

Figure 16: Muscle response as predicted by the present model and the model of Spyrou et al. [54] for FVF $=90 \%$ and $G^{(1)} / G^{(2)}=20$ under (a) in-plane tension, (b) longitudinal shear, (c) in-plane shear, and (d) perpendicular shear.

model presented in Section 2 can be actually employed to perform simulations of 3D organ-level muscle geometries using the numerical implementation scheme proposed in this paper.

The fusiform muscle is modeled using an axisymmetric geometry (Fig. 17a) where the local orientations of the fibers in the undeformed configuration (i.e. the unit vectors $\mathbf{m}_{0}$ in Eq. 22) are determined by interpolation between the corresponding directions of the fiber on the axis of symmetry and the outermost fibers on the free surface of the muscle (see also Spyrou [51]). The fusiform muscle is subjected to tensile loading under active $\left(0<f_{a} \leq 1\right)$ or passive $\left(f_{a}=0\right)$ conditions. In particular, the one muscle end is fixed, whereas at the other end a uniform displacement is applied and the corresponding axial reaction force is determined.

On the other hand, the pennate muscle is modeled using a simple parallelepiped geometry (Fig. 18a) where the muscle fibers are oriented at an angle of $45 \mathrm{deg}$ relative to the vertical z-axis. The pennate muscle is analyzed under perpendicular shear loading conditions while being active $\left(0<f_{a} \leq 1\right)$ or passive $\left(f_{a}=0\right)$. This is achieved by fixing the one muscle end, applying at the other end a uniform horizontal displacement in the x-direction as shown in Fig. 18a, and calculating the corresponding horizontal reaction force.

The finite element calculations are carried out in Abaqus using eight-node hexahedral hybrid elements, quasi-static conditions, and finite strains. The local directions of the muscle fibers are defined as initial conditions at all Gauss integration points in terms of the local unit vectors $\mathbf{m}_{0}$. When the activity of the muscle is taken into account the activation function $f_{a}$ increases linearly from 0 to 1 over a time period of $1 \mathrm{~s}$ while the whole load is applied over the same time period. In both applications the multiscale constitutive 


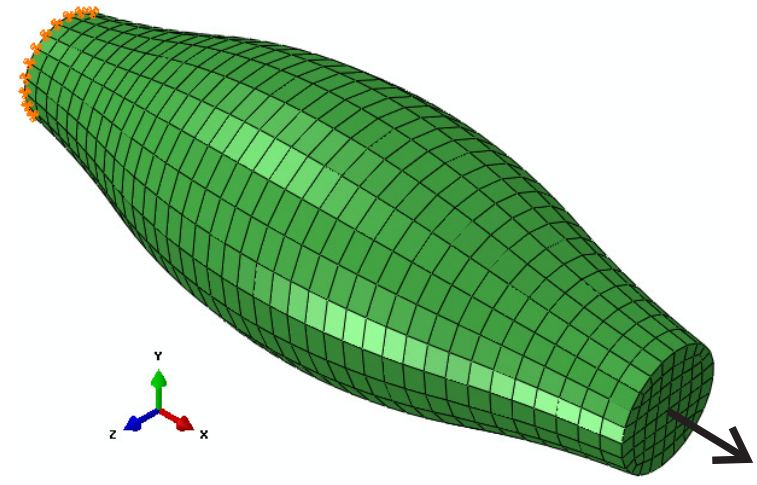

(a)

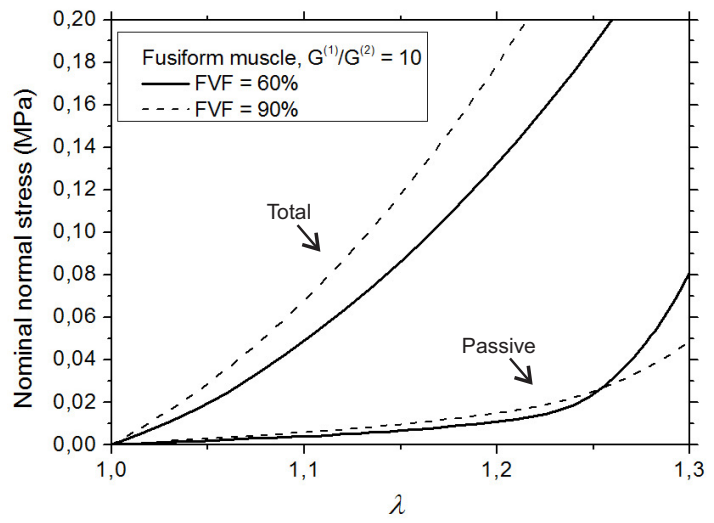

(b)

Figure 17: (a) FE mesh and boundary conditions of an idealized fusiform muscle geometry. The initial muscle length is 220 $\mathrm{mm}$ and the cross sectional diameters at the ends of the muscle and at the middle of the muscle belly are $36 \mathrm{~mm}$ and $80 \mathrm{~mm}$ respectively. (b) Average stress/stretch total and passive muscle response under axial tensile loading for $\mathrm{FVF}=90 \%$ and $\mathrm{FVF}$ $=60 \%$.

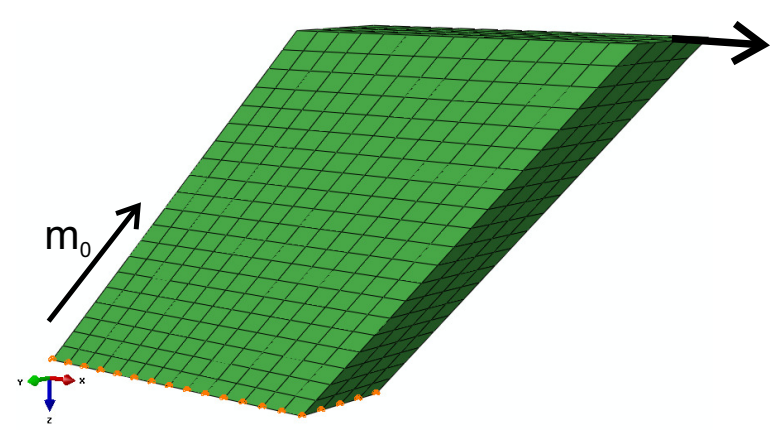

(a)

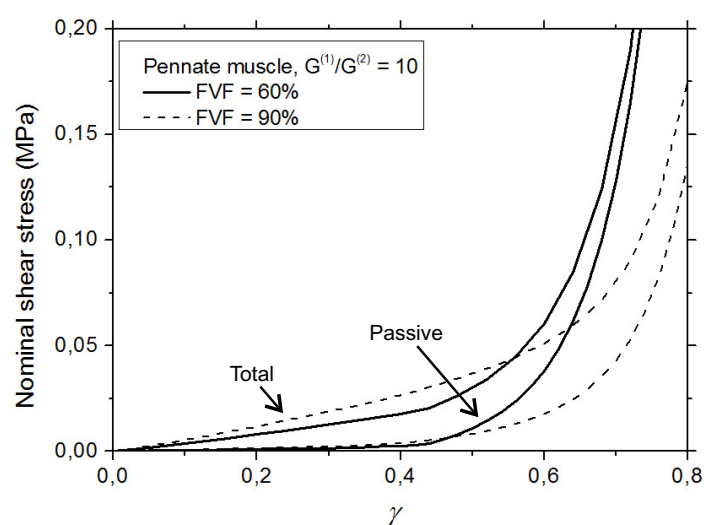

(b)

Figure 18: (a) FE mesh and boundary conditions of an idealized pennate muscle geometry. The initial muscle height, width, and depth are $200 \mathrm{~mm}, 200 \mathrm{~mm}$, and $60 \mathrm{~mm}$ respectively. (b) Average stress/strain total and passive muscle response under perpendicular shear loading conditions for $\mathrm{FVF}=90 \%$ and $\mathrm{FVF}=60 \%$.

model presented in Section 2 is used to describe the mechanical behavior of muscle tissues. We assume that FVF in the muscles is either $60 \%$ denoting a pathologic case or $90 \%$ indicating a healthy tissue. Also, the shear moduli contrast between muscle fibers and ECM is taken as $G^{(1)} / G^{(2)}=10$. The values for the parameters $\dot{\lambda}_{m}^{\min }, k_{c}, k_{e}, d$ that enter the active stress-strain rate function $f_{r}$ in Eq. (11) are taken from Böl and Reese [4] as $\dot{\lambda}_{m}^{\min }=-17 s^{-1}, k_{c}=5, k_{e}=5, d=1.5$. The remaining values of the model parameters used in this analysis are the same with those used in Section 4.2.

Figure 17b shows the average nominal normal stress/stretch response for the fusiform muscle and Figure $18 \mathrm{~b}$ shows the average nominal shear stress/strain response for the pennate muscle. In both cases, the average nominal stress is found by dividing the calculated reaction force with the surface area at which the corresponding displacement has been imposed. For the fusiform muscle, the average axial stretch $\lambda$ is defined as the ratio between the final and the initial muscle lengths. For the pennate muscle, the average nominal strain $\gamma$ is defined as the ratio between the applied horizontal displacement and the initial vertical muscle height.

The fusiform muscle response follows the trend of the corresponding response under loading in the muscle 
fiber direction presented in Fig. 8. In particular, when muscle remains passive $\left(f_{a}=0\right)$ the higher FVF leads to higher stresses at lower stretches, whereas at higher stretches a cross-over of the response occurs due to the increased load-bearing capacity of the ECM. On the other hand, when muscle activity is taken into account $\left(f_{a}>0\right)$ the response is dominated by the behavior of the muscle fibers for $\lambda \leq 1.3$. Accordingly, a similar trend can be observed for the pennate muscle response, i.e. the overall muscle response is controlled by the muscle fibers when shear strains are maintained under a certain threshold $(\simeq 0.5-0.6$ in this case $)$ whereas above this threshold the effective response becomes dominated by ECM's behavior.

\section{Concluding remarks}

In this work, we have developed analytical and numerical homogenization-based models that may be used to describe the mechanical behavior of skeletal muscles at finite strains subjected to three-dimensional loading conditions. The analytical model incorporates muscle's microstructural characteristics including volume fractions, material properties, and spatial distribution of its constituents, namely muscle fibers and ECM. In order to achieve this goal an approximate decoupled homogenization scheme has been used to bridge the different length scales of the muscle structure. Specifically, the analytical model considers that the effective isotropic and anisotropic parts resulting from the corresponding isotropic and anisotropic response of the constituents can be decoupled. This allows to first homogenize the anisotropic response of the muscle using an exact Voigt homogenization scheme. In turn, for the isotropic part, we use a recently developed model for two-phase fiber reinforced composites by López Jímenez [30] and extend it in the context of muscle fibers embedded in an extracellular matrix (ECM). The two contributions are then added in the current configuration and are shown to deliver very accurate estimates when compared with the numerical homogenization model which is resolved via full field finite element (FE) simulations. To the best knowledge of the authors, this is the first analytical micromechanics-based model in the literature which is able to describe fairly accurately the mechanical behavior of skeletal muscles upon the full range of three-dimensional loadings for a wide range of different compositions and material properties.

In addition, a numerical homogenization model based on eroded Voronoi tesselations has been developed in order to perform virtual tests using computational micromechanics techniques. This framework allowed the calculation of the effective properties of an idealized composite muscle by analyzing numerically the mechanical response of a unit cell (i.e. a representative volume element-RVE) of the microstructure under various loading conditions. In this paper, motivated by optical image observations of the muscle fiberECM microstructure, an approach based on eroded Voronoi tesselation models, where the Voronoi polygons represent the muscle fibers and the finite thickness regions surrounding the polygons represent the ECM, was chosen as a suitable and in the same time efficient way to represent realistically skeletal muscle's microstructural cross sections.

The analytical model parameters were identified using experimental data from the literature whereas the full field finite element calculations of the periodic Voronoi unit cells were used to assess the accuracy of the analytical homogenized muscle model presented in this study. At first, the analytical model is shown to be able to probe accurately (by proper identification of the material parameters) the experimentally measured muscle response under different loading conditions. Secondly, the analytical model is assessed by comparison with the full field numerical results and is found to be extremely accurate for a wide range of fiber volume fractions (FVF from $60 \%$ to $90 \%$ ), different material contrasts (shear modulus contrast $G^{(1)} / G^{(2)}=5-50$ ), and the full range of loading conditions. Since significant microstructural variations may occur among healthy and diseased muscle tissues, it is important that a reliable constitutive model for muscle tissue can describe muscle behavior accurately within a vast range of FVF, loading conditions, and different material properties of the constituents.

As opposed to phenomenological modeling, the proposed microstructural modeling approach takes into account explicitly the dependence of the macroscopic response of the muscle upon the underlying microstructural arrangement, micro-deformation mechanisms, and material properties of its constituents. The developed models allow to explain the relation between muscle microstructure and macroscopic mechanical properties, as well as to make predictions about muscle behavior upon microstructural changes. Since physiological muscular diseases may cause significant microstructural changes, such models can become important 
in better understanding muscle function and the fundamental role of mechanics under such conditions. For example, the model is able to explain analytically the experimentally observed significant role of intramuscular connective tissue in the increased muscle stiffness of a spastic muscle, as discussed in Section 4.2. Such observations may lead to new therapeutic treatments for skeletal muscle disease-related dysfunctions.

In the context of the finite element method, the proposed analytical muscle model has been numerically implemented in a very efficient user-material subroutine which, in turn, allows for the simulation of three-dimensional muscle geometries of any complexity. This is critical for biological muscular systems since it provides the opportunity to investigate physiological- or disease-related problems in a multiscale framework via computer simulations in real time. In this paper, for illustration purposes, the model has been applied successfully to simulate the mechanical response of idealized fusiform and pennate muscle structures under different microstructural conditions. In particular, the effect of different muscle composition on the macroscopic mechanical response of muscles of different type has been examined.

In this study the ECM's behavior was considered as transversely isotropic with all collagen fibrils aligned in one direction. Although endomysium (and perimysium) show preferred orientations with respect to the muscle fiber direction (see Section 2.1.2), normally these connective tissues contain networks of fibers whose orientations are dispersed around that preferred orientations. Thus, for a more realistic material description, the model for ECM can be substituted by any other model that takes into account its internal microstructure, i.e., the presence of a fibril network at a much smaller length scale than that of the muscle fiber itself, and consider the dispersion of the collagen fibrils around a mean (preferred) orientation $\theta$ with respect to the muscle fiber direction $\mathbf{m}_{0}$ by following either the so-called "angular integration" method originated by Lanir [25] or the "generalized structure tensor" approach formulated by Gasser et al. [11].

In closing, it should be remarked that both the numerical and the analytical models proposed in this work involve the assumption that the muscle-fiber and ECM phases are initially perfectly bonded and remain perfectly bonded throughout any given loading. The computational micromechanics framework presented in this work could be a perfect means to investigate numerically the effect of imperfect phase bonding, as may occur due to local damage of the transmembrane connections of the phases, by modifying appropriately the rigid FE mesh connections between muscle constituents [e.g. 62]. This aspect will be examined in a future work. Also, the model used at the local scale for the muscle fiber constituent do not account for several features, such as different fiber types (fast- or slow-twitch), grouping of fibers in motor units, fiber recruitment, muscle fatigue, and muscle residual force enhancement [6]. Such features could be readily included in the current version of the homogenization model by appropriate modifications of equation (8). The addition of such features in the muscle fiber constituent model will be considered in a future work. An additional study that would be of interest and is not carried out here is the cyclic response of muscles and the resulting dissipation $[44,39,61]$. Such an analysis would have been straightforward in the present numerical setting by taking into account the viscoelastic characteristics of the constituents' passive behavior. Finally, the present study strongly suggests that the muscle fiber volume fraction plays a critical role upon the overall muscle response and further experiments in that direction could prove very useful to address those effects in a more quantitative manner.

\section{Acknowledgments}

K.D. would like to acknowledge partial support from the European Research Council (ERC) under the European Unions Horizon 2020 research and innovation program (Grant Agreement No. 636903).

\section{Appendix A. Numerical implementation of the analytical model}

In this appendix we describe the numerical implementation of the analytical constitutive model in the context of the finite element method. In view of the non-linearity and the rate dependence of the model, the finite element solution is developed incrementally. The constitutive calculations are carried out at the element Gauss integration points. In particular, at a given Gauss point, the directional vector in the reference 
configuration $\mathbf{m}_{0}$, the homogenized solution $\left(\widehat{\boldsymbol{F}}_{n}, \widehat{\boldsymbol{\sigma}}_{n},\left.\widehat{\lambda}_{m}\right|_{n}\right.$ at time $t_{n}$ as well as the average deformation gradient $\widehat{\boldsymbol{F}}_{n+1}$ at time $t_{n+1}=t_{n}+\Delta t$ are known, and the problem is to determine $\left(\widehat{\boldsymbol{\sigma}}_{n+1},\left.\widehat{\lambda}_{m}\right|_{n+1}, \widehat{\mathbf{m}}_{n+1}\right)$.

The constitutive calculations at the Gauss points are carried out in the following order:

$$
\begin{aligned}
& \widehat{J}_{n+1}=\operatorname{det} \widehat{\boldsymbol{F}}_{n+1}, \\
& \overline{\widehat{\boldsymbol{F}}}_{n+1}=\left(\widehat{J}_{n+1}\right)^{-1 / 3} \widehat{\boldsymbol{F}}_{n+1}, \\
& \overline{\boldsymbol{B}}_{n+1}=\overline{\widehat{\boldsymbol{F}}}_{n+1} \cdot \overline{\widehat{\boldsymbol{F}}}_{n+1}^{T}, \\
& \widehat{\boldsymbol{B}}_{n+1}=\widehat{\boldsymbol{F}}_{n+1} \cdot \widehat{\boldsymbol{F}}_{n+1}^{T}, \\
& \widehat{\boldsymbol{C}}_{n+1}=\widehat{\boldsymbol{F}}_{n+1}^{T} \cdot \widehat{\boldsymbol{F}}_{n+1}, \\
& \left.\widehat{\widehat{I}}_{1}\right|_{n+1}=\operatorname{tr}\left(\widehat{\boldsymbol{B}}_{n+1}\right) \text {, } \\
& \left.\widehat{I}_{4}\right|_{n+1}=\mathbf{m}_{0} \cdot \widehat{\boldsymbol{C}}_{n+1} \cdot \mathbf{m}_{0}, \\
& \left.\widehat{I}_{5}\right|_{n+1}=\mathbf{m}_{0} \cdot \widehat{C}_{n+1}^{2} \cdot \mathbf{m}_{0}, \\
& \widehat{\mathbf{m}}_{n+1}=\frac{1}{\left|\widehat{\boldsymbol{F}}_{n+1} \cdot \mathbf{m}_{0}\right|} \widehat{\boldsymbol{F}}_{n+1} \cdot \mathbf{m}_{0}, \\
& \left.\widehat{\lambda}_{m}\right|_{n+1}=\sqrt{\mathbf{m}_{0} \cdot \widehat{\boldsymbol{C}}_{n+1} \cdot \mathbf{m}_{0}}, \\
& \left.\dot{\hat{\lambda}}_{m}\right|_{n+1}=\frac{\left.\widehat{\lambda}_{m}\right|_{n+1}-\left.\widehat{\lambda}_{m}\right|_{n}}{\Delta t}, \\
& \widehat{K}=c \cdot K^{(1)}+(1-c) \cdot K^{(2)}, \\
& G_{I H}=(1-c)^{2}\left(1+\frac{2(2-c) c}{(1-c)^{2}} \frac{G^{(1)}}{G^{(2)}}+\frac{G^{(1)^{2}}}{G^{(2)^{2}}}\right) \frac{G^{(2)}}{2}- \\
& -(1-c)^{2} \frac{G^{(1)}-G^{(2)}}{2} \sqrt{\frac{2}{(1-c)^{2}} \frac{G^{(1)}}{G^{(2)}}+\left(1+\frac{2(2-c) c}{(1-c)^{2}} \frac{G^{(1)}}{G^{(2)}}+\frac{G^{(1)^{2}}}{G^{(2)^{2}}}\right)} \text {, } \\
& G_{n}=c G^{(1)}+(1-c) G^{(2)}, \\
& G_{H S}=\frac{(1-c) G^{(2)}+(1+c) G^{(1)}}{(1+c) G^{(2)}+(1-c) G^{(1)}} G^{(2)},
\end{aligned}
$$




$$
\begin{aligned}
&\left.\widehat{\boldsymbol{\sigma}}_{i}\right|_{n+1}=\widehat{K}\left(\widehat{J}_{n+1}-1\right) \boldsymbol{\delta}+\frac{G_{I H}}{\widehat{J}_{n+1}}\left(\widehat{\widehat{\boldsymbol{B}}}_{n+1}-\frac{1}{3} \widehat{I}_{1} \boldsymbol{\delta}\right) \\
&+\frac{1}{\widehat{J}_{n+1}}\left(\frac{G_{I H}-G_{n}}{\sqrt{\left.\widehat{I}_{4}\right|_{n+1}}}+\frac{\left.\left(G_{I H}-G_{H S}\right) \widehat{I}_{5}\right|_{n+1}}{\left.\widehat{I}_{4}\right|_{n+1}}+\left.\widehat{I}_{4}\right|_{n+1}\left(G_{n}-G_{H S}\right)\right) \widehat{\mathbf{m}}_{n+1} \widehat{\mathbf{m}}_{n+1} \\
& \quad-\frac{G_{I H}-G_{H S}}{\widehat{J}_{n+1}}\left(\widehat{\mathbf{m}}_{n+1} \widehat{\boldsymbol{B}}_{n+1} \cdot \widehat{\mathbf{m}}_{n+1}+\widehat{\mathbf{m}}_{n+1} \cdot \widehat{\boldsymbol{B}}_{n+1} \widehat{\mathbf{m}}_{n+1}\right) \\
&\left.\sigma_{0}^{(1)}\right|_{n+1}=\left.\sigma_{\max } f_{a}\right|_{n+1} f_{e}\left(\left.\widehat{\lambda}_{m}\right|_{n+1}\right) f_{r}\left(\left.\dot{\widehat{\lambda}}_{m}\right|_{n+1}\right)+f_{p}^{(1)}\left(\left.\widehat{\lambda}_{m}\right|_{n+1}\right), \\
&\left.\sigma_{0}^{(2)}\right|_{n+1}=f_{p}^{(2)}\left(\left.\widehat{\lambda}_{m}\right|_{n+1}, \theta_{0}\right), \\
&\left.\widehat{\sigma}_{0}\right|_{n+1}=\left.c \cdot \sigma_{0}^{(1)}\right|_{n+1}+\left.(1-c) \cdot \sigma_{0}^{(2)}\right|_{n+1}, \\
& \widehat{\sigma}_{n+1}=\left.\left.\widehat{\lambda}_{m}\right|_{n+1} \widehat{\sigma}_{0}\right|_{n+1}, \\
&\left.\widehat{\boldsymbol{\sigma}}_{a}\right|_{n+1}=\widehat{\sigma}_{n+1} \widehat{\mathbf{m}}_{n+1} \widehat{\mathbf{m}}_{n+1}, \\
&\left.\widehat{\widehat{\boldsymbol{\sigma}}}\right|_{n+1}=\left.\widehat{\boldsymbol{\sigma}}_{i}\right|_{n+1}+\left.\widehat{\boldsymbol{\sigma}}_{a}\right|_{n+1} .
\end{aligned}
$$

The "implicit" version of ABAQUS is used, in which the finite element formulation is based on the weak form of the equilibrium equations, the solution is carried out incrementally, and the discretized nonlinear equations are solved by using Newton's method. The Jacobian of the equilibrium Newton-loop requires the so-called "linearization moduli" of the algorithm that handles the constitutive equations; these moduli are defined in terms of a fourth-order tensor $\widehat{\mathcal{C}}$ that relates the variation of stress to the variation of strain over the increment. Because the Jaumann objective stress rate is used in the "implicit" version of ABAQUS for continuum elements, in order to determine the fourth-order tensor $\widehat{\mathcal{C}}$ we need to define a relationship of the form

$$
\stackrel{\nabla}{\widehat{\sigma}}=\widehat{\mathcal{C}}: \widehat{\boldsymbol{D}}=\left(\widehat{\mathcal{C}}^{i}+\widehat{\mathcal{C}}^{a}\right): \widehat{\boldsymbol{D}}
$$

where $\stackrel{\nabla}{\widehat{\sigma}}=\dot{\hat{\boldsymbol{\sigma}}}-\widehat{\boldsymbol{W}} \cdot \widehat{\boldsymbol{\sigma}}-\widehat{\boldsymbol{\sigma}} \cdot \widehat{\boldsymbol{W}}^{T}$ is the Jaumann rate of the average Cauchy stress tensor, $\widehat{\boldsymbol{D}}$ the average deformation rate tensor, and $\widehat{\boldsymbol{W}}$ the average spin tensor.

\section{Evaluation of $\widehat{\mathcal{C}}^{a}$}

The details of the derivation of the anisotropic part of $\widehat{\mathcal{C}}$ which corresponds to $\widehat{\mathcal{C}}^{a}$ are reported in Spyrou [51]. Here we note that

$$
\widehat{\mathcal{C}}_{i j k l}^{a}=\widehat{\lambda}_{m}\left(\widehat{\sigma}_{0}+D\right) H_{i j k l}+\widehat{\sigma} A_{i j k l},
$$

with

$$
\begin{gathered}
D=\widehat{\lambda}_{m}\left(\frac{\partial \widehat{\sigma}_{0}}{\partial \widehat{\lambda}_{m}}+\frac{1}{\Delta t} \frac{\partial \widehat{\sigma}_{0}}{\partial \dot{\hat{\lambda}}_{m}}\right), \\
H_{i j k l}=\widehat{m}_{i} \widehat{m}_{j} \widehat{m}_{k} \widehat{m}_{l}, \\
A_{i j k l}=\frac{1}{2}\left(\delta_{i k} \widehat{m}_{j}+\delta_{j k} \widehat{m}_{i}\right) \widehat{m}_{l}+\frac{1}{2}\left(\delta_{i l} \widehat{m}_{j}+\delta_{j l} \widehat{m}_{i}\right) \widehat{m}_{k}-2 H_{i j k l} .
\end{gathered}
$$




\section{Evaluation of $\widehat{\mathcal{C}}^{i}$}

The effective behavior derived by the homogenization of the constituents' isotropic behavior is characterized by the stored-energy function $\widehat{W}_{I H}$. The elasticity tensor in the material description $\overline{\mathcal{C}}$ relates the work conjugate pairs of stress and strain tensors and measures the change in stress which results from a change in strain under consideration:

$$
\partial \widehat{\boldsymbol{S}}=\overline{\mathcal{C}}: \partial \widehat{\boldsymbol{E}}
$$

where $\widehat{\boldsymbol{S}}$ is the average 2nd Piola-Kirchhoff stress tensor and $\widehat{\boldsymbol{E}}$ is the average Green-Lagrange strain tensor. Taking into account that $\widehat{\boldsymbol{S}}$ can be derived from $\widehat{W}_{I H}$ according to $\widehat{\boldsymbol{S}}=2 \partial \widehat{W}_{I H} / \partial \widehat{\boldsymbol{C}}$ the elasticity tensor $\overline{\mathcal{C}}$ can be found by

$$
\overline{\mathcal{C}}=4 \frac{\partial^{2} \widehat{W}_{I H}}{\partial \widehat{\boldsymbol{C}} \partial \widehat{\boldsymbol{C}}}=4 \frac{\partial}{\partial \widehat{\boldsymbol{C}}}\left(\frac{\partial \widehat{W}_{I H}}{\partial \widehat{\widehat{I}}_{1}} \frac{\partial{\widehat{\widehat{I}_{1}}}}{\partial \widehat{\boldsymbol{C}}}+\frac{\partial \widehat{W}_{I H}}{\partial \widehat{I}_{4}} \frac{\partial \widehat{I}_{4}}{\partial \widehat{\boldsymbol{C}}}+\frac{\partial \widehat{W}_{I H}}{\partial \widehat{I}_{5}} \frac{\partial \widehat{I}_{5}}{\partial \widehat{\boldsymbol{C}}}+\frac{\partial \widehat{W}_{I H}}{\partial \widehat{J}} \frac{\partial \widehat{J}}{\partial \widehat{\boldsymbol{C}}}\right)
$$

Using (24) and (64) with the product and chain rules, we arrive at a closed-form expression for $\overline{\mathcal{C}}$

$$
\begin{aligned}
\overline{\mathcal{C}}_{i j k l} & =\widehat{K} \widehat{J}\left[(2 \widehat{J}-1) \widehat{C}_{i j}^{-1} \widehat{C}_{k l}^{-1}+2(\widehat{J}-1) \frac{\partial \widehat{C}_{i j}^{-1}}{\partial \widehat{C}_{k l}}\right]+\frac{2 G_{I H} \widehat{J}^{-2 / 3}}{3}\left(-\delta_{i j} \widehat{C}_{k l}^{-1}-\widehat{C}_{i j}^{-1} \delta_{k l}\right) \\
& +\frac{2 G_{I H} \widehat{\widehat{I}}_{1}}{3}\left(-\frac{\partial \widehat{C}_{i j}^{-1}}{\partial \widehat{C}_{k l}}+\frac{1}{3} \widehat{C}_{i j}^{-1} \widehat{C}_{k l}^{-1}\right)-\left[\frac{3\left(G_{I H}-G_{n}\right)}{\widehat{I}_{4}^{5 / 2}}+\frac{4\left(G_{I H}-G_{H S}\right) \widehat{I}_{5}}{\widehat{I}_{4}^{3}}\right] m_{i}^{0} m_{j}^{0} m_{k}^{0} m_{l}^{0} \\
& +\frac{2\left(G_{I H}-G_{H S}\right)}{\widehat{I}_{4}^{2}} m_{i}^{0} m_{j}^{0} m_{k}^{0} \widehat{C}_{l p} m_{p}^{0}+\frac{2\left(G_{I H}-G_{H S}\right)}{\widehat{I}_{4}^{2}} m_{i}^{0} m_{j}^{0} m_{e}^{0} \widehat{C}_{e k} m_{l}^{0}+\frac{2\left(G_{I H}-G_{H S}\right)}{\widehat{I}_{4}^{2}} m_{i}^{0} \widehat{C}_{j p} m_{p}^{0} m_{k}^{0} m_{l}^{0} \\
& -\frac{2\left(G_{I H}-G_{H S}\right)}{\widehat{I}_{4}} m_{i}^{0} I_{j p k l} m_{p}^{0}+\frac{2\left(G_{I H}-G_{H S}\right)}{\widehat{I}_{4}^{2}} m_{e}^{0} \widehat{C}_{e i} m_{j}^{0} m_{k}^{0} m_{l}^{0}-\frac{2\left(G_{I H}-G_{H S}\right)}{\widehat{I}_{4}} m_{e}^{0} I_{e i k l} m_{j}^{0}
\end{aligned}
$$

where $\frac{\partial \widehat{C}_{i j}^{-1}}{\partial \widehat{C}_{k l}}=-\frac{1}{2}\left(\widehat{C}_{i k}^{-1} \widehat{C}_{j l}^{-1}+\widehat{C}_{i l}^{-1} \widehat{C}_{j k}^{-1}\right)$ and $I_{i j k l}=\frac{1}{2}\left(\delta_{i k} \delta_{j l}+\delta_{i l} \delta_{j k}\right)$.

The spatial representation of (63) can be shown to be (e.g. [16])

$$
\dot{\hat{\boldsymbol{\tau}}}-\widehat{\boldsymbol{L}} \cdot \widehat{\boldsymbol{\tau}}-\widehat{\boldsymbol{\tau}} \cdot \widehat{\boldsymbol{L}}^{T}=\widehat{J} \widetilde{\mathcal{C}}: \widehat{\boldsymbol{D}}
$$

where $\widehat{\boldsymbol{\tau}}=\widehat{J} \widehat{\boldsymbol{\sigma}}$ is the average Kirchhoff stress tensor, $\widehat{\boldsymbol{L}}=\widehat{\boldsymbol{D}}+\widehat{\boldsymbol{W}}$ is the average velocity gradient with $\widehat{\boldsymbol{D}}$ the average deformation rate tensor and $\widehat{\boldsymbol{W}}$ the average spin tensor, and $\widetilde{\mathcal{C}}$ is the spatial description of the elasticity tensor defined as the push-forward operation of $\overline{\mathcal{C}}$ times a factor of $\widehat{J}^{-1}$

$$
\widetilde{\mathcal{C}}_{i j k l}=\frac{1}{\widehat{J}} \widehat{F}_{i I} \widehat{F}_{j J} \widehat{F}_{k K} \widehat{F}_{l L} \overline{\mathcal{C}}_{I J K L}
$$

Using (65) and (67) a closed-form expression for $\widetilde{\mathcal{C}}_{i j k l}$ with index notation reads

$$
\begin{aligned}
& \widetilde{\mathcal{C}}_{i j k l}=\widehat{K}(2 \widehat{J}-1) \delta_{i j} \delta_{k l}-\widehat{K}(\widehat{J}-1)\left(\delta_{i k} \delta_{j l}+\delta_{i l} \delta_{j k}\right)-\frac{2 G_{I H}}{3 \widehat{J}}\left(\widehat{\widehat{B}}_{i j} \delta_{k l}+\delta_{i j} \widehat{\widehat{B}}_{k l}\right) \\
& +\frac{2 G_{I H} \widehat{\widehat{I}_{1}}}{3 \widehat{J}}\left(\frac{1}{2}\left(\delta_{i k} \delta_{j l}+\delta_{i l} \delta_{j k}\right)+\frac{1}{3} \delta_{i j} \delta_{k l}\right)-\frac{1}{\widehat{J}}\left[\frac{3\left(G_{I H}-G_{n}\right)}{\widehat{I}_{4}^{1 / 2}}+\frac{4\left(G_{I H}-G_{H S}\right) \widehat{I}_{5}}{\widehat{I}_{4}}\right] \widehat{m}_{i} \widehat{m}_{j} \widehat{m}_{k} \widehat{m}_{l} \\
& +\frac{2}{\widehat{J}}\left(G_{I H}-G_{H S}\right) \widehat{m}_{i} \widehat{m}_{j} \widehat{m}_{t} \widehat{B}_{t k} \widehat{m}_{l}-\frac{2}{\widehat{J}}\left(G_{I H}-G_{H S}\right)\left[-\widehat{m}_{i} \widehat{B}_{j t} \widehat{m}_{t} \widehat{m}_{k} \widehat{m}_{l}+\frac{1}{2}\left(\widehat{m}_{i} \widehat{B}_{j k} \widehat{m}_{l}+\widehat{m}_{i} \widehat{B}_{j l} \widehat{m}_{k}\right)\right] \\
& -\frac{2}{\widehat{J}}\left(G_{I H}-G_{H S}\right)\left[-\widehat{m}_{t} \widehat{B}_{t i} \widehat{m}_{j} \widehat{m}_{k} \widehat{m}_{l}+\frac{1}{2}\left(\widehat{m}_{k} \widehat{B}_{i l} \widehat{m}_{j}+\widehat{m}_{l} \widehat{B}_{i k} \widehat{m}_{j}\right)\right]+\frac{2}{\widehat{J}}\left(G_{I H}-G_{H S}\right) \widehat{m}_{i} \widehat{m}_{j} \widehat{m}_{k} \widehat{B}_{l t} \widehat{m}_{t} .
\end{aligned}
$$


By substituting $\widehat{\boldsymbol{L}}=\widehat{\boldsymbol{D}}+\widehat{\boldsymbol{W}}$ in Eq.(66) we arrive at an expression of the form

$$
{\stackrel{\nabla}{\widehat{\sigma}_{i}}}_{i}=\widehat{\mathcal{C}}^{i}: \widehat{\boldsymbol{D}}
$$

where

$$
\widehat{\mathcal{C}}_{i j k l}^{i}=\widetilde{\mathcal{C}}_{i j k l}+\frac{1}{2}\left(\delta_{i k} \widehat{\sigma}_{j l}+\widehat{\sigma}_{i k} \delta_{j l}+\delta_{i l} \widehat{\sigma}_{j k}+\widehat{\sigma}_{i l} \delta_{j k}\right)
$$

A closed-form expression for $\widehat{\mathcal{C}}_{i j k l}^{i}$ reads

$$
\begin{aligned}
& \widehat{\mathcal{C}}_{i j k l}^{i}=\widehat{K}(2 \widehat{J}-1) \delta_{i j} \delta_{k l}+\frac{G_{I H}}{\widehat{J}}\left[\frac{1}{2}\left(\delta_{i k} \widehat{\widehat{B}}_{j l}+\widehat{\widehat{B}}_{i k} \delta_{j l}+\delta_{i l} \widehat{\widehat{B}}_{j k}+\overline{\widehat{B}}_{i l} \delta_{j k}\right)-\frac{2}{3} \widehat{\widehat{B}}_{i j} \delta_{k l}-\frac{2}{3} \delta_{i j} \widehat{\widehat{B}}_{k l}+\frac{2 \widehat{I}_{1}}{9} \delta_{i j} \delta_{k l}\right] \\
& +\frac{1}{2 \widehat{J}}\left(\frac{G_{I H}-G_{n}}{\widehat{I}_{4}^{1 / 2}}+\frac{\left(G_{I H}-G_{H S}\right) \widehat{I}_{5}}{\widehat{I}_{4}}+\widehat{I}_{4}\left(G_{n}-G_{H S}\right)\right)\left(\delta_{i k} \widehat{m}_{j} \widehat{m}_{l}+\widehat{m}_{i} \widehat{m}_{k} \delta_{j l}+\delta_{i l} \widehat{m}_{j} \widehat{m}_{k}+\widehat{m}_{i} \widehat{m}_{l} \delta_{j k}\right) \\
& -\frac{G_{I H}-G_{H S}}{2 \widehat{J}}\left(\delta_{i k} \widehat{m}_{j} \widehat{B}_{l t} \widehat{m}_{t}+\delta_{i k} \widehat{m}_{t} \widehat{B}_{t j} \widehat{m}_{l}+\widehat{m}_{i} \widehat{B}_{k t} \widehat{m}_{t} \delta_{j l}+\widehat{m}_{t} \widehat{B}_{t i} \widehat{m}_{k} \delta_{j l}\right) \\
& -\frac{G_{I H}-G_{H S}}{2 \widehat{J}}\left(\widehat{m}_{i} \widehat{B}_{l t} \widehat{m}_{t} \delta_{j k}+\widehat{m}_{t} \widehat{B}_{t i} \widehat{m}_{l} \delta_{j k}+\delta_{i l} \widehat{m}_{j} \widehat{B}_{k t} \widehat{m}_{t}+\delta_{i l} \widehat{m}_{t} \widehat{B}_{t j} \widehat{m}_{k}\right) \\
& -\frac{1}{\widehat{J}}\left[\frac{3\left(G_{I H}-G_{n}\right)}{\widehat{I}_{4}^{1 / 2}}+\frac{4\left(G_{I H}-G_{H S}\right) \widehat{I}_{5}}{\widehat{I}_{4}}\right] \widehat{m}_{i} \widehat{m}_{j} \widehat{m}_{k} \widehat{m}_{l} \\
& -\frac{2}{\widehat{J}}\left(G_{I H}-G_{H S}\right)\left[-\widehat{m}_{t} \widehat{B}_{t i} \widehat{m}_{j} \widehat{m}_{k} \widehat{m}_{l}+\frac{1}{2}\left(\widehat{m}_{k} \widehat{B}_{i l} \widehat{m}_{j}+\widehat{m}_{l} \widehat{B}_{i k} \widehat{m}_{j}\right)\right] \\
& +\frac{2}{\widehat{J}}\left(G_{I H}-G_{H S}\right)\left[\widehat{m}_{i} \widehat{m}_{j} \widehat{m}_{k} \widehat{B}_{l t} \widehat{m}_{t}+\widehat{m}_{i} \widehat{m}_{j} \widehat{m}_{t} \widehat{B}_{t k} \widehat{m}_{l}\right] \\
& -\frac{2}{\widehat{J}}\left(G_{I H}-G_{H S}\right)\left[-\widehat{m}_{i} \widehat{B}_{j t} \widehat{m}_{t} \widehat{m}_{k} \widehat{m}_{l}+\frac{1}{2}\left(\widehat{m}_{i} \widehat{B}_{j k} \widehat{m}_{l}+\widehat{m}_{i} \widehat{B}_{j l} \widehat{m}_{k}\right)\right]
\end{aligned}
$$

In equations 59 and 71 all quantities are evaluated at the end of the increment under consideration, i.e., at $t=t_{n+1}$. It is noted that the fourth-order tensor $\widehat{\mathcal{C}}$ has both the "minor" and "major" symmetries, i.e.,

$$
\widehat{\mathcal{C}}_{i j k l}=\widehat{\mathcal{C}}_{j i k l}=\widehat{\mathcal{C}}_{i j l k}=\widehat{\mathcal{C}}_{k l i j} .
$$

\section{References}

[1] ABAQUS, 2013. Dassault Systems Abaqus. ABAQUS/Standard Version 6.13, Simulia Corp.

[2] Agoras, M., Lopez-Pamies, O., Castaneda, P.P., 2009. A general hyperelastic model for incompressible fiber-reinforced elastomers. J. Mech. Phys. Solids 57, 268-286.

[3] Blemker, S.S., Pinsky, P.M., Delp, S.L., 2005. A 3D model of muscle reveals the causes of nonuniform strains in the biceps brachii. J. Biomech. 38, 657-665.

[4] Böl, M., Reese, S., 2008. Micromechanical modelling of skeletal muscles based on the finite element method. Comput. Methods Biomech. Biomed. Engin. 11, 489-504.

[5] Ceelen, K.K., Oomens, C.W.J., Baaijens, F.P.T., 2008. Microstructural analysis of deformation-induced hypoxic damage in skeletal muscle. Biomech. Model. Mechanobiol. 7, 277-284.

[6] Chapelle, D., Le Tallec, P., Moireau, P., Sorine, M.. 2012. An energy-preserving muscle tissue model: formulation and compatible discretizations. Int. J. Multiscale Comp. Eng., Begell House, 10 (2), pp.189-211.

[7] deBotton G., 2005. Transversely isotropic sequentially laminated composites in finite elasticity. J Mech Phys Solids 53, $1334-61$.

[8] deBotton G, Hariton I, Socolsky E., 2006. Neo-hookean fiber-reinforced composites in finite elasticity. J Mech Phys Solids 54, 533-59.

[9] Destrade, M., Horgan, C.O., Murphy, J.G., 2015. Dominant negative Poynting effect in simple shearing of soft tissues. J. Eng. Math. 95, 87-98.

[10] Ehret, A.E., Böl, M., Itskov, M., 2011. A continuum constitutive model for the active behaviour of skeletal muscle. J. Mech. Phys. Solids 59, 625-636. 
[11] Gasser, T.C., Ogden, R.W., Holzapfel, G.A., 2006. Hyperelastic modelling of arterial layers with distributed collagen fiber orientations. J. R. Soc. Interface 3, 15-35.

[12] Geuzaine, C., Remacle, J.-F., 2009. Gmsh: A 3-D finite element mesh generator with built-in pre- and post-processing facilities. Int. J. Num. Meth. Eng. 79(11), 1309-1331.

[13] Hawkins, D., Bey, M., 1994. A comprehensive approach for studying muscle-tendon mechanics. J. Biomech. Eng. 116, $51-55$.

[14] Heidlauf, T., Röhrle, O., 2014. A multiscale chemo-electro-mechanical skeletal muscle model to analyze muscle contraction and force generation for different muscle fiber arrangements. Front. Physiol. 5, 498/1-14.

[15] Hill, R., 1972. On constitutive macro-variables for heterogeneous solids at finite strain. Proc. R. Soc. A 326, 131-147.

[16] Holzapfel, G.A., 2000. Nonlinear solid mechanics: a continuum approach for engineering. Wiley.

[17] Holzapfel, G.A., Gasser, T.C., Ogden, R.W., 2000. A new constitutive framework for arterial wall mechanics and a comparative study of material models. J. Elasticity 61, 1-48.

[18] Holzapfel, G.A., Schulze-Bauer, C.A.J., Feigl, G., Regitnig, P., 2005. Single lamellar mechanics of the human lumbar anulus fibrosus. Biomech. Model. Mechanobiol. 3, 125-140.

[19] Holzapfel, G.A., Ogden, R.W., 2015. On the tension-compression switch in soft fibrous solids. Eur. J. Mech. A-Solid 49, 561-569.

[20] Huijing, P.A., 1999. Muscle as a collagen fiber reinforced composite: a review of force transmission in muscle and whole limb. J. Biomech. 32, 329-345.

[21] Javili, A., Chatzigeorgiou, G., Steinmann, P., 2013. Computational homogenization in magneto-mechanics. Int. J. Solids. Struct. 50, 4197-4216.

[22] Jozsa, L., Thöring, J., Järvinen, M., Kannus, P., Lehto, M., Kvist, M., 1988. Quantitative alterations in intramuscular connective tissue following immobilization: an experimental study in the rat calf muscles. Exp. Mol. Pathol. 49, $267-278$.

[23] Kanit, T., Forest, S., Galliet, I., Mounoury, V., Jeulin, D., 2003. Determination of the size of the representative volume element for random composites: statistical and numerical approach. Int. J. Sol. Struct. 40, 3647-3679.

[24] Kjær, M., 2004. Role of extracellular matrix in adaptation of tendon and skeletal muscle to mechanical loading. Physiol. Rev. 84, 649-698.

[25] Lanir, Y., 1983. Constitutive equations for fibrous connective tissues. J. Biomech. 16, 1-12.

[26] Lemos, R.R., Epstein, M., Herzog, W., Wyvill, B., 2004. A framework for structured modeling of skeletal muscle. Comput. Methods Biomech. Biomed. Engin. 7, 305-317.

[27] Lieber, R.L., Bodine-Fowler, S.C., 1993. Skeletal muscle mechanics: implications for rehabilitation. Phys. Ther. 73, $844-856$.

[28] Lieber, R.L., Runesson, E., Einarsson, F., Fridén, J., 2003. Inferior mechanical properties of spastic muscle bundles due to hypertrophic but compromised extracellular matrix material. Muscle Nerve 28, 464-471.

[29] Lieber, R.L., Fridén, J., 2004. Implications of muscle design on surgical reconstruction of upper extremities. Clin. Orthop. Relat. Res. 419, 267-279.

[30] López Jímenez, F., 2014. Modeling of soft composites under three-dimensional loading. Compos. Part B Eng. 59, $173-180$.

[31] Lopez-Pamies O. 2010. An exact result for the macroscopic response of particle reinforced Neo-Hookean solids. J. Appl. Mech. 77, 021016/1-5.

[32] Lopez-Pamies O, Idiart MI. 2010. Fiber-reinforced hyperelastic solids: a realizable homogenization constitutive theory. J. Eng. Math. 68, 57-83.

[33] Lopez-Pamies O., Idiart M., Li Z. 2011. On microstructure evolution in fiber-reinforced elastomers and implications for their mechanical response and stability. J Eng. Mat. Tech. 133, 011007/1-10.

[34] Lopez-Pamies, O., Goudarzi, T., Danas, K., 2013. The nonlinear elastic response of suspensions of rigid inclusions in rubber. II. A simple explicit approximation for finite-concentration suspensions. J. Mech. Phys. Solids 61, 19-37.

[35] Marcucci, L., Reggiani, C., Natali, A.N., Pavan, P.G., 2017. From single muscle fiber to whole muscle mechanics: a finite element model of a muscle bundle with fast and slow fibers. Biomech. Model. Mechanobiol. 16, 1833-1843.

[36] Mbiakop, A., Constantinescu, A., Danas, K., 2015. An analytical model for porous single crystals with ellipsoidal voids. J. Mech. Phys. Solids 84, 436-467.

[37] Meyer, G.A., Lieber, R.L., 2011. Elucidation of extracellular matrix mechanics from muscle fibers and fiber bundles. J. Biomech. 44, 771-773.

[38] Michel, J.C., Moulinec, H., Suquet, P., 1999. Effective properties of composite material with periodic microstructure: a computational approach. Comput. Methods Appl. Mech. Eng. 172, 109-143.

[39] Minajeva, A., Kulke, M., Fernandez, J.M., Linke, W.A., 2001. Unfolding of titin domains explains the viscoelastic behavior of skeletal myofibrils. Biophys. J. 80, 1442-1451.

[40] Morrow, D.A., Haut Donahue, T.L., Odegard, G.M., Kaufman, K.R., 2010. Transversely isotropic tensile material properties of skeletal muscle tissue. J. Mech. Behav. Biomed. Mater. 3, 124-129.

[41] Ogden, R.W., 1974. On the overall moduli of non-linear elastic composite materials. J. Mech. Phys. Solids 22, 541-553.

[42] Purslow, P.P., 1989 Strain-induced reorientation of an intramuscular connective tissue network: implications for passive muscle elasticity. J. Biomech. 22, 21-31.

[43] Purslow, P.P., Trotter, J.A., 1994. The morphology and mechanical properties endomysium in series-fibred muscles: variations with muscle length. J. Muscle Res. Cell. Motil. 15, 299-308.

[44] Purslow, P.P., Wess, T.J., Hukins, D.W., 1998. Collagen orientation and molecular spacing during creep and stressrelaxation in soft connective tissues. J. Exp. Biol. 201, 135-142.

[45] Rassier, D.E., Lee, E.J., Herzo, W., 2005. Modulation of passive force in single skeletal muscle fibres. Biol. Lett. 1, 
$342-345$.

[46] Roche, J.A., Tulapurkar, M.E., Mueller, A.L., van Rooijen, N., Hasday, J.D., Lovering, R.M., Bloch, R.J., 2015. Myofiber damage precedes macrophage infiltration after in vivo injury in dysferlin-deficient A/J mouse skeletal muscle. Am. J. Pathol. 185, 1686-1698.

[47] Röhrle, O., Pullan, A.J., 2007. Three-dimensional finite element modelling of muscle forces during mastication. J. Biomech. 40, 3363-3372.

[48] Sharafi, B., Blemker, S.S., 2010. A micromechanical model of skeletal muscle to explore the effects of fiber and fascicle geometry. J. Biomech. 43, 3207-3213.

[49] Sharafi, B., Blemker, S.S., 2011. A mathematical model of force transmission from intrafascicularly terminating muscle fibers. J. Biomech. 44, 2031-2039.

[50] Smith, L.R., Lee, K.S., Ward, S.R., Chambers, H.G., Lieber, R.L., 2011. Hamstring contractures in children with spastic cerebral palsy result from a stiffer extracellular matrix and increased in vivo sarcomere length. J. Physiol. 589, $2625-2639$.

[51] Spyrou, L.A., 2009. Muscle and tendon tissues: constitutive modeling, numerical implementation and applications. Dissertation, University of Thessaly.

[52] Spyrou, L.A., Aravas, N., 2011. Muscle and tendon tissues: constitutive modeling and computational issues. J. Appl. Mech. 78, 041015/1-10.

[53] Spyrou, L.A., Aravas, N., 2012. Muscle-driven finite element simulation of human foot movements. Comput. Methods Biomech. Biomed. Engin. 15, 925-934.

[54] Spyrou, L.A., Agoras, M., Danas, K., 2017. A homogenization model of the Voigt type for skeletal muscle. J. Theor. Biol. 414, 50-61.

[55] Thacker, B.E., Tomiya, A., Hulst, J.B., Suzuki, K.P., Bremner, S.N., Gastwirt, R.F., Greaser, M.L., Lieber, R.L., Ward, S.R., 2012. Passive mechanical properties and related proteins change with botulinum neurotoxin A injection of normal skeletal muscle. J. Orthop. Res. 30, 497-502.

[56] Tidball, J.G., Wehling-Henricks, M., 2004. Evolving therapeutic strategies for Duchenne muscular dystrophy: targeting downstream events. Pediatr. Res. 56, 831-841.

[57] Torquato, S., 2002. Random Heterogeneous Materials. Springer, New York.

[58] Trotter, J.A., Richmond, F.J.R., Purslow, P.P., 1995. Functional morphology and motor control of series-fibered muscles. Exerc. Sport Sci. Rev. 23, 167-214.

[59] Van Leeuwen, J.L., Kier, W.M., 1997. Functional design of tentacles in squid: linking sarcomere ultrastructure to gross morphological dynamics. Philos. Trans. R. Soc. Lond. B Biol. Sci. 352, 551-571.

[60] Van Loocke, M., Lyons, C.G., Simms, C.K., 2006. A validated model of passive muscle in compression. J. Biomech. 39, 2999-3009.

[61] Van Loocke, M., Lyons, C.G., Simms, C.K., 2008. Viscoelastic properties of passive skeletal muscle in compression: Stress-relaxation behaviour and constitutive modelling. J. Biomech. 41, 1555-1566.

[62] Virgilio, K.M., Martin, K.S., Peirce, S.M., Blemker, S.S., 2015. Multiscale models of skeletal muscle reveal the complex effects of muscular dystrophy on tissue mechanics and damage susceptibility. Interface Focus 5, 20140080/1-10.

[63] Wein, R., 2007. Exact and approximate construction of offset polygons. Computer-Aided Design 39(6), 518-527.

[64] Yan, D.M., Wang, K., Lévy, B., Alonso, L., 2011. Computing 2D Periodic Centroidal Voronoi Tessellation. Proceedings of the Eighth International Symposium on Voronoi Diagrams in Science and Engineering (ISVD), $177-184$.

[65] Zajac, F.E., 1989. Muscle and tendon: properties, models, scaling and application to biomechanics and motor control. Crit. Rev. Biomed. Eng. 17, 359-411. 